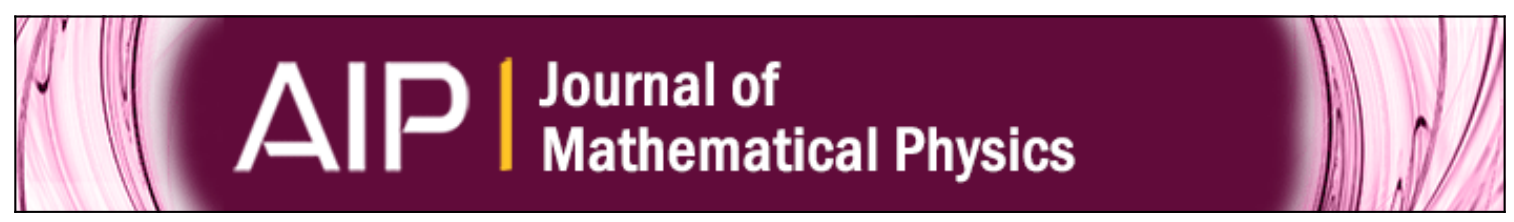

\title{
Inverse scattering on the line for a Dirac system
}

D. B. Hinton, A. K. Jordan, M. Klaus, and J. K. Shaw

Citation: Journal of Mathematical Physics 32, 3015 (1991); doi: 10.1063/1.529046

View online: http://dx.doi.org/10.1063/1.529046

View Table of Contents: http://scitation.aip.org/content/aip/journal/jmp/32/11 ?ver=pdfcov

Published by the AIP Publishing

\section{AAlP Re-register for Table of Content Alerts}




\section{Inverse scattering on the line for a Dirac system}

D. B. Hinton

Department of Mathematics, University of Tennessee, Knoxville, Tennessee 37996

A. K. Jordan

Center for Advanced Space Sensing, Code 4210, US Naval Research Laboratory, Washington, DC 20375

M. Klaus and J. K. Shaw

Department of Mathematics, Virginia Tech, Blacksburg, Virginia 24061

(Received 8 May 1991; accepted for publication 2 July 1991)

The whole-line version of the Gelfand-Levitan-Marchenko (GLM) equation for a Dirac system is studied. A new derivation of the GLM equation is given, under weaker hypotheses than Frolov's earlier treatment [Sov. Math. Dokl. 13, 1468 (1972)], and the complete inversion is carried out in some explicit cases in which a spectral gap is present. Previous calculations of this type are restricted either to a scalar potential or degenerate gap. Applications are discussed in connection with optical couplers and soliton equations.

\section{INTRODUCTION}

Dirac systems are two-component first-order systems of ordinary differential equations in the unknown

$$
y=\left(\begin{array}{l}
y_{1} \\
y_{2}
\end{array}\right)
$$

of the form

$$
\begin{aligned}
& J y^{\prime}=\left(\begin{array}{cc}
\lambda+v_{2}(x)-c & p(x) \\
p(x) & \lambda+v_{1}(x)+c
\end{array}\right) y, \\
& J=\left(\begin{array}{cc}
0 & -1 \\
1 & 0
\end{array}\right),
\end{aligned}
$$

where $c>0$ is a constant and $\lambda$ is the spectral parameter. A prime denotes ordinary differentiation. A complete scattering theory of (1.1) exists ${ }^{1-6}$ in which $p(x), v_{1}(x)$, and $v_{2}(x)$ are suitably small at $\pm \infty$, resulting in a spectral gap $(-c, c)$, i.e., the spectrum is discrete in $(-c, c)$ and continuous elsewhere. The definitions and properties of scattering quantities are given in Sec. II.

The origin of (1.1) is the Dirac equation in $\mathbb{R}^{3}$ given by ${ }^{7}$

$$
-c \sum_{j=1}^{3} \alpha_{j} \frac{\partial \psi}{\partial x_{j}}+\left(V(x)-\alpha_{4} m c^{2}\right) \psi=E \psi,
$$

which physically describes a relativistic electron in an electrostatic field $V(x)$. In (1.2) $\psi$ is the four-component wavefunction, $\alpha_{j}$ are the Pauli spin matrices, $m$ is the particle mass, $E$ is the energy, and $c$ is the speed of light. If $V(x)$ is spherically symmetric, then (1.2) is spectrally equivalent to the more familiar system

$$
J y^{\prime}+\left(\begin{array}{cc}
V(x)-m c / h & k / x \\
k / x & V(x)+m c / h
\end{array}\right) y=\frac{\lambda}{h c} y,
$$

where $k$ (integer) and $h$ are constants. The separation of variables leading from (1.2) to (1.3) is given in Ref. 7. In order to shed more light on purely spectral questions most modern investigations have placed (1.3) into the more general context (1.1); this is the point of view taken here.

Note in (1.3) that $v_{1}=v_{2}$ for the general system (1.1). For the purposes of inverse scattering, however, the case $v_{1}=v_{2}$ contains an inherit nonuniqueness, as will be explained in Sec. II. In fact, the morc usual assumption is $v_{1}=-v_{2}=v$, so that (1.1) becomes

$$
J y^{\prime}=\left(\begin{array}{cc}
\lambda-v(x)-c & p(x) \\
p(x) & \lambda+v(x)+c
\end{array}\right) y .
$$

System (1.4) has been associated with inverse scattering for nonlinear evolution equations and with certain waveguide problems.

We are concerned with the inverse scattering theory associated with the whole-line version of $(1.4),-\infty<x<\infty$, and specifically with two of its featurcs. The first is the fundamental Gelfand-Levitan-Marchenko (GLM) equation (Sec. III), which relates scattering data to the potential terms $p(x)$ and $v(x)$, and the second is the machinery by which the GLM equation is actually solved (Sec. IV). Fro$\operatorname{lov}^{8}$ is usually credited with having derived the GLM equation for (1.4) on the full line. Frolov assumes, for differentiable $v(x)$ and $p(x)$, the power law growth

$$
|v(x)| \leqslant C /(1+|x|)^{2+\epsilon}, \quad|p(x)| \leqslant C /(1+|x|)^{1+\epsilon},
$$

where $C>0$ and $\epsilon>0$ (arbitrary) are constants; however, his paper contains no proofs. Asano and Kato ${ }^{9,10}$ have established forward and inverse scattering theories for a non-selfadjoint version of (1.4). Assuming, also, differentiable potentials, Asano and Kato derive the GLM equation by relying on a Parseval relation. The setting of Asano and Kato is very abstract, and it seems that simple proofs of the existence of transformation operators and the GLM equation, based on the ideas in Refs. 11 and 12, would be useful. We provide these proofs in Secs. II and III for system (1.4) under the following weaker assumptions that remove the differentiability hypotheses and allow some singular behavior of $p$ and $v$. Let $L^{1}$ denote the usual Lebesque class on $(-\infty, \infty)$ and let $L_{1}^{1}=\left\{h \in L^{1} \mid(1+|x|) h(x) \in L^{1}\right\} ; L_{1}^{1}$ is sometimes called the Faddeev class. For $h \in L_{1}^{1}$ let 


$$
\begin{aligned}
& \sigma_{h}(x)=\int_{x}^{\infty}|h(t)| d t, \\
& \sigma_{h}^{(1)}(x)=\int_{x}^{\infty}(1+|t|)|h(t)| d t .
\end{aligned}
$$

For system (1.4) we now assume that $p$ and $v$ are locally integrable and that there exists $f \in L_{1}^{1} \cap L^{2}$ such that for all $x$,

$$
\begin{aligned}
& f(x) \geqslant 0, \quad f(x)=f(-x), \\
& |v(x)| \leqslant f(x), \\
& |p(x)| \leqslant(1+|x|) f(x), \quad|p( \pm x)| \leqslant \sigma_{f}(x), \\
& \int_{x}^{\infty} f(t)^{2} d t \leqslant\left(\sigma_{f}(x)\right)^{2} .
\end{aligned}
$$

Note that $v(x)$ is allowed to have $L^{2}$-type singularities, but that $p(x)$ is bounded. The power law assumptions of Fro$10 v^{8}$ are more restrictive than (1.6)-(1.9). We will make an additional assumption about reflection coefficients later.

Having the GLM equation in hand we take the simplest explicit case, namely, the rational reflection coefficient, and ask whether there is a machinery leading to a solution for $p$ and $v$. Surprisingly, there do not appear to be any results in the literature that solve the inverse problem for (1.1) with rational scattering data and also with a nondegenerate spectral gap. As we shall see in Sec. IV, taking $c=0$ in (1.1) simplifies the entire problem enormously. Our second contribution thus comes in Sec. IV, where we solve a prototype inverse problem with a rational reflection coefficient and nontrivial gap. Our method is adapted from an analogous algorithm for the Schrödinger equation suggested originally by Kay and Moses ${ }^{13,1}$ and carried out further by Jordan ${ }^{14}$ for purposes of the design of optical waveguides.

The outline of this paper is as follows. In Sec. II we briefly review the literature, attempt to motivate the paper by indicating applications, give definitions, and prove the existence of transformation operators. In Sec. III we derive the GLM equation, and we close the paper in Sec. IV with the complete inversion of prototype problems generated by rational reflection coefficients.

\section{BACKGROUND AND PRELIMINARIES}

It was pointed out by Gasymov and Levitan ${ }^{15,16}$ that inverse problems for the fully general system (1.1) are not uniquely solvable whether on the half-line or full line; instead, there are classes within which certain inverse scattering problems have only one solution. To describe the classes we assume first that $p, v_{i} \in L^{1}$ and recall that for $\operatorname{Im}(\lambda)>0$ there exist Jost solutions $f^{( \pm)}(x, \lambda)$ of (1.1) which obey the asymptotics

$$
\begin{aligned}
& f^{( \pm \prime}(x, \lambda) e^{\mp i w x} \rightarrow\left(\begin{array}{c} 
\pm(\lambda+c) / i w) \\
1
\end{array}\right), \\
& x \rightarrow \pm \infty, w=\sqrt{\lambda^{2}-c^{2}} .
\end{aligned}
$$

The existence of Jost solutions holds under much weaker hypotheses than (1.6)-(1.9), ${ }^{1-3,5}$ but the stronger conditions (1.6)-(1.9) will be needed for the existence of transformation operators ${ }^{12}$ of (1.1), which we discuss presently. The Jost functions of (1.1) are defined by ${ }^{3}$

$$
F^{( \pm)}(\lambda)=f_{\mathrm{I}}^{( \pm)}(0, \lambda), \quad \operatorname{Im}(\lambda)>0,
$$

where the subscript 1 denotes the first component. The socalled $S$ matrix, or scattering matrix, is the scalar quantity given by

$$
S(\lambda)=\overline{F^{(+)}(\lambda)} / F^{(+)}(\lambda),
$$

where the overbar represents the complex conjugate. This is the main scattering quantity for (1.1) on the half-line $0 \leqslant x<\infty$ (one can also define $S^{(-)}=\overline{F^{(-)}} / F^{(-)}$and work on $-\infty<x \leqslant 0$ ) and corresponds to the analogous $S$ matrix for the Schrödinger operator. Now we introduce for $0 \leqslant x<\infty$ the orthogonal transformation ${ }^{5,17} y(x)$ $=H(x) z(x)$, where

$$
H(x)=\left(\begin{array}{ll}
\cos W(x) & -\sin W(x) \\
\sin W(x) & \cos W(x)
\end{array}\right)
$$

and $W(x)=\int_{x}^{\infty}\left(v_{1}+v_{2}\right) / 2$, so that the $z(x)$ system is of the form

$$
J z^{\prime}=\left(\begin{array}{cc}
\lambda-\tilde{v}(x)-c & \tilde{p}(x) \\
\tilde{p}(x) & \lambda+\tilde{v}(x)+c
\end{array}\right) z,
$$

that is, of the form (1.1) with $v_{2}=-v_{1}$. Moreover, if

$$
\int_{0}^{\infty} \frac{v_{1}+v_{2}}{2}=2 n \pi, n \text { an integer, }
$$

then (1.1) and (2.4) have the same Jost function because of (2.3) and $y(0)=z(0)$; the same holds for $S(\lambda)$. The correspondence from $S(\lambda)$ back to (1.1) must therefore be inherently nonunique unless (1.1) and (2.4) coincide, which they in fact do if $v_{1}=-v_{2}$. Indeed, Gasymov ${ }^{18}$ has given (for a $2 n$-component system, actually) necessary and sufficient conditions on $S(\lambda)$ such that it should be the scattering matrix of a unique (half-line) potential in the class (1.5) with $v_{2}=-v_{1}$ (see, also, Ref. 9). This justifies in some sense working within the general restriction $v_{2}=-v_{1}$. Gasymov and Levitan ${ }^{15}$ go on to characterize the spectral function $\rho(\lambda)$ for the half-line problem (1.1) under $v_{2}=-v_{1}$. The same authors ${ }^{16}$ also characterize the scattering phase $\delta(\lambda)$ under the same restriction and assuming also (1.5). On the other hand, Weiss et al. ${ }^{19}$ considered the case $v_{1}=v_{2}$ and $p(x)$ singular on $(0, \infty)$ and derived the GLM equation for this situation by means of an integral equation that contains a singular term. The present authors ${ }^{6}$ have recently shown existence of the transformation operator and derived the GLM equation for the general system (1.1) on the half-line under (1.5) and (2.5).

Frolov ${ }^{8}$ also considered the restricted case $v_{2}=-v_{1}$ on $-\infty<x<\infty$ under condition (1.5) in establishing the equation for the transformation kernel of (1.1). Frolov displays the GLM equation for the kernel, but gives no proof of its derivation. We will prove existence of the transformation operator and give our own derivation of the GLM equation (Sec. III) for the restricted case $v_{2}=-v_{1}$ of (1.4) under (1.6) $-(1.9)$. We give the definitions of the reflection coeffcient, transformation operator, GLM equation, etc. below.

Turning to the physical motivation for (1.4) we recall the method of Zakharov and Shabat, ${ }^{20,21}$ which obtains (soliton) solutions of $i u_{t}+u_{x x}+c|u|^{2} u=0$ by solving the inverse scattering problem for the Dirac system 
$u_{1}^{\prime}=i \lambda u_{1}+q(x) u_{2}, u_{2}^{\prime}=-i \lambda u_{2}+\overline{q(x)} u_{1}$, a system that is equivalent to (1.1) under the transformation

$$
u=2^{-1 / 2}\left(\begin{array}{cc}
1 & -i \\
-i & 1
\end{array}\right) y
$$

and in which $q(x)=c+v_{1}(x)+i p(x)$ and $v_{2}=-v_{1}$. The first paper ${ }^{20}$ takes $q(x) \rightarrow 0$, which means that a spectral gap is absent, but a gap appears if one allows $q(x)$ to have asymptotic limits at $\pm \infty .{ }^{21}$ Reference 21 is not a rigorous treatment; instead, it connects the nonlinear evolution equation to the Dirac system and proceeds to derive the Marchenko equation on a heuristic basis. For example, no specific hypothesis is placed on the reflection coefficient $r(k)$, although it is necessary to have $r(k)=O(1 / k)$; see (2.17) and the proof of Theorem 3.1 below. Zakharov and Shabat then focus their discussion on the reflectionless case $r(k)=0$, which is the pure soliton case, and proceed to describe a two-soliton interaction.

Grosse $^{22}$ notes that solitons are similarly associated with a system

$$
\psi^{\prime}=\left(\begin{array}{cc}
u(x) & v(x)+\lambda \\
v(x)-\lambda & -u(x)
\end{array}\right) \psi, \quad-\infty<x<\infty,
$$

but he allows $v(x)$ to have asymptotic limits at $\pm \infty$. This makes the above equivalent to (1.4) with $v_{2}=-v_{1}$ and $c \neq 0$. Grosse ${ }^{22}$ performs an explicit inversion of the system, with a nondegenerate gap $(c \neq 0)$, but only in the reflectionless case (reflection coefficient $=0$ ). In a related paper, Grosse and Opelt ${ }^{23}$ give another explicit (reflectionless) inversion based on a separation ansatz for the transformation kernel.

Recently Bava et al. ${ }^{24,25}$ have discussed a system of the Zakharov and Shabat type in connection with optical couplers: They begin with the system

$$
\begin{aligned}
& \frac{d a_{1}}{d z}+i \beta_{1} a_{1}=i h_{11}(z) a_{1}+h_{12}(z) a_{2}, \\
& \frac{d a_{2}}{d z}+i \beta_{2} a_{2}=i h_{22}(z) a_{2}+h_{21}(z) a_{1},
\end{aligned}
$$

which is used to model the coupling between two waveguide modes with the propagation constants $\beta_{1}$ and $\beta_{2}$. The $a_{j}$ are complex modal amplitudes. Two waveguides with, say, their fundamental modes excited are brought into physical proximity so that an ensemble field is formed. If $\psi_{1}$ and $\psi_{2}$ are the separate fields in isolation, then coupling theory asserts that the ensemble field may be written as

$$
\psi(x, z)=a_{1}(z) \psi_{1}(x)+a_{2}(z) \psi_{2}(x),
$$

where time dependence is suppressed and the guide is taken to be planar so that there is no $y$ dependence. The transverse axis is $x$ and the longitudinal axis is $z$, so that (2.7) represents the interchange of power along the $z$ axis of the fibers. The terms $h_{i j}(z)$ are called coupling coefficients. ${ }^{26}$

In Refs. 24 and 25 the system (2.6) is transformed to the form

$i \frac{d b_{1}}{d z}-i Q(z) b_{2}=k b_{1}, \quad i \frac{d b_{2}}{d z}+i R(z) b_{1}=k b_{2}$,

which is of the Zakharov and Shabat type, and then a program is presented for inverting (2.8) [i.e., solving for $Q(z)$ and $R(z)$ ] when given a rational reflection coefficient; it is implicitly assumed that $Q, R \rightarrow 0$, i.e., no gap is present.

It should be noted, however, that (2.6) is not the optical coupler model that is normally used. The self-coupling terms $h_{11}(z)$ and $h_{22}(z)$ are an order of magnitude smaller ${ }^{26}$ than the cross-coupling terms $h_{12}(z)$ and $h_{21}(z)$ and so are usually ignored. Furthermore, the cross-coupling terms should be allowed to be complex and are nearly constant. Based on these observations we are led to introduce in (2.6) $u_{1}(z)=e^{i k z} e^{i \beta_{1} z} a_{1} \quad$ and $\quad u_{2}(z)=e^{-i k z} e^{i \beta_{2} z} a_{2} \quad$ with $k=\left(\beta_{2}-\beta_{1}\right) / 2$. Then the $u$ system is

$$
u^{\prime}=\left(\begin{array}{cc}
i k+i h_{11} & h_{12} \\
h_{21} & -i k+i h_{22}
\end{array}\right) u \text {. }
$$

Putting

$$
M=\left(\begin{array}{ll}
1 & i \\
i & 1
\end{array}\right)
$$

and $y=M u$ we obtain

$$
y^{\prime}=\frac{1}{2}\left(\begin{array}{cc}
i\left(h_{11}+h_{22}+h_{21}-h_{12}\right) & 2 k+h_{11}-h_{22}+h_{21}+h_{12} \\
-2 k+h_{22}-h_{11}+h_{21}+h_{12} & i\left(h_{11}+h_{22}-h_{21}+h_{12}\right)
\end{array}\right) y .
$$

We now postulate that $h_{12}$ and $h_{21}$ are small, complex-valued perturbations around a constant value

$h_{12}(z)=c+u(z)-i v(z), \quad h_{21}(z)=c+u(z)+i v(z)$, and if we incorporate these into the previous equation and drop the self-coupling terms $h_{11}(z)$ and $h_{22}(z)$ we obtain

$$
\left(\begin{array}{cc}
0 & -1 \\
1 & 0
\end{array}\right) y^{\prime}=k y+\left(\begin{array}{cc}
-c-u(z) & v(z) \\
v(z) & c+u(z)
\end{array}\right) y,
$$

which is the Dirac system (1.4) with $k$ as the spectral parameter. The inverse problem then amounts physically to solving for the cross-coupling coefficients $h_{12}$ and $h_{21}$.

Before looking at the GLM equation we give a discussion of transformation operators and scattering coefficients.
For the rest of the paper we assume the reduced form of (1.1), namely, the system

$J y^{\prime}=\left(\begin{array}{cc}-v(x)-c & p(x) \\ p(x) & v(x)+c\end{array}\right) y+\lambda y, \quad-\infty<x<\infty$,

$J=\left(\begin{array}{cc}0 & -1 \\ 1 & 0\end{array}\right)$

and where (unless stated otherwise) $v(x)$ and $p(x)$ are locally $L^{2}$ and satisfy conditions $(1.6)-(1.9)$. If $v(x)=p(x)=0$, then (2.9) has constant coefficients and among its solutions are the free Jost solutions 


$$
f_{0}^{( \pm)}(x, \lambda)=e^{ \pm i w x}\left(\begin{array}{c} 
\pm(\lambda+c) / i w \\
1
\end{array}\right)
$$

where $w=\sqrt{\lambda^{2}-c^{2}}$. The branch of $w(\lambda)$ is chosen so that $w(\lambda)>0$ for real $\lambda>c$ and $w(\lambda)<0$ for $\lambda<-c$; in particu$\operatorname{lar}, \operatorname{Im}(w)>0$ iff $\operatorname{Im}(\lambda)>0$ and $\overline{w(\lambda)}=-w(\bar{\lambda})$.

The free and perturbed Jost solutions (2.1) are connected through the transformation kernels $K^{( \pm)}(x, t)$ by means of

$f^{(+)}(x, \lambda)=f_{0}^{(+)}(x, \lambda)+\int_{x}^{\infty} K^{(+)}(x, t) f_{0}^{(+)}(t, \lambda) d t$

$$
\begin{aligned}
f^{(-)}(x, \lambda)= & f_{0}^{(-)}(x, \lambda) \\
& +\int_{-\infty}^{x} K^{(-)}(x, t) f_{0}^{(-)}(t, \lambda) d t, \lambda \neq \pm c .
\end{aligned}
$$

Existence of the kernels for differentiable potentials has been proved by Gasymov ${ }^{18}$ under conditions (1.5), by Asano and Kato, ${ }^{9}$ and by Faddeev and Takhtajan ${ }^{27}$ for potentials in the Schwartz ("rapidly decreasing") class. Here we give a separate proof under conditions (1.6)-(1.9). We give the proof only for $K^{(+)}(x, t)$; a similar result holds for $(2.12)$.

Theorem 2.1: Suppose that $v(x)$ and $p(x)$ satisfy (1.6)(1.9). Let $\sigma_{f}(x)=\int_{x}^{\infty} f(t) d t, \quad \sigma_{f}^{(1)}(x)=\int_{x}^{\infty}(1+|t|)$ $\times f(t) d t$, and $\alpha(x)=1+\max (0,-x)$. Then a function $K^{(+)}(x, t)$ exists for $t \geqslant x$ such that (2.11) holds and such that

$$
\begin{aligned}
& \left|K_{11}^{(+)}(x, t)\right|,\left|K_{22}^{(+)}(x, t)\right| \\
& \quad \leqslant c_{1}\left[\exp \left(c_{2} \alpha(x) \sigma_{f}^{(1)}(x)\right) \sigma_{f}((x+t) / 2)\right],
\end{aligned}
$$

$$
\begin{aligned}
& \left|K_{12}^{(+)}(x, t)\right|,\left|K_{21}^{(+)}(x, t)\right| \\
& \leqslant c_{1}[f(x+t) / 2)+\exp \left(c_{2} \alpha(x) \sigma_{f}^{(1)}(x)\right) \\
& \left.\quad \times \sigma_{f}((x+t) / 2) /(|x|+1)\right],
\end{aligned}
$$

where $c_{1}$ and $c_{2}$ are constants. The kernel $K^{(+)}$satisfies the initial conditions

$\left(\begin{array}{cc}-v(x) & p(x) \\ p(x) & v(x)\end{array}\right)=K^{(+)}(x, x) J-J K^{(+)}(x, x)$

and is the uniform (in $x) L^{1}$ limit of kernels $K_{n}^{(+)}(x, t)$,

$$
\lim _{n \rightarrow \infty} \int_{x}^{\infty}\left|K_{n}^{(+)}(x, t)-K^{(+)}(x, t)\right| d t=0,
$$

that satisfies the partial differential equation (PDE)

$$
\begin{aligned}
& J \frac{\partial}{\partial x} K_{n}^{(+)}(x, t) \\
& \quad-\left(\begin{array}{cc}
-v_{n}(x)-c & p_{n}(x) \\
p_{n}(x) & v_{n}(x)+c
\end{array}\right) K_{n}^{(+)}(x, t) \\
& \quad+\frac{\partial}{\partial t} K_{n}^{(+)}(x, t) J+K_{n}^{(+\gamma}(x, t)\left(\begin{array}{cc}
-c & 0 \\
0 & c
\end{array}\right)=0,
\end{aligned}
$$

where $v_{n}(x)$ and $p_{n}(x)$ are smooth and compactly supported potentials which converge to $v(x)$ and $p(x)$ in the $L^{1}$ norm.

Before we give the proof of Theorem 2.1 we recall some notation from Gasymov ${ }^{18}$ and prove two lemmas. Following Gasymov, ${ }^{18}$ we work with the following integral equation. Let

$$
\begin{aligned}
& \Omega(x)=\left(\begin{array}{cc}
-v(x) & p(x) \\
p(x) & v(x)
\end{array}\right), \\
& D(x)=\left(\begin{array}{cc}
-\frac{1}{2} p(x)+c \int_{x}^{\infty} v(t) d t & -\frac{1}{2} v(x) \\
-\frac{1}{2} v(x) & -\frac{1}{2} p(x)+c \int_{x}^{\infty} v(t) d t
\end{array}\right)
\end{aligned}
$$

and consider the following integral equation for an unknown function $A(x, t)$ :

$$
\begin{aligned}
A(x, t)= & D\left(\frac{x+t}{2}\right) \\
& -c \int_{x}^{(x+t) / 2} \int_{t+x-s}^{t-x+s}\left(\begin{array}{cc}
-v(s) A_{11}(s, u)+p(s) A_{21}(s, u) & 0 \\
0 & -p(s) A_{12}(s, u)-v(s) A_{22}(s, u)
\end{array}\right) d u d s \\
& -c \int_{(x+t) / 2}^{\infty} \int_{s}^{t-x+s}\left(\begin{array}{cc}
-v(s) A_{11}(s, u)+p(s) A_{21}(s, u) & 0 \\
0 & -p(s) A_{12}(s, u)-v(s) A_{22}(s, u)
\end{array}\right) d u d s \\
& -\frac{1}{2} \int_{x}^{\infty} \Omega(s)(J A(s, t-x+s)-A(s, t-x+s) J) d s \\
& -\frac{1}{2} \int_{x}^{(x+t) / 2} \Omega(s)(J A(s, t+x-s)+A(s, t+x-s) J) d s .
\end{aligned}
$$

The solution of (2.17) will yield the transformation kernel $A=K^{(+)}$, but the proof is unfortunately quite complicated and will be given in several stages. We will first show that $(2.17)$ has a solution $A(x, t)$ that satisfies the bounds (2.13) and (2.14). Then we will construct a sequence $K_{n}^{(+)}(x, t)$ of kernels that are associated with smooth and compactly supported potentials $p_{n} \rightarrow p, v_{n} \rightarrow p$ which satisfy (2.16). Following Gasymov (who assumes absolutely continuous $p$ and $v$ ) it then follows that $K_{n}^{(+)}$satisfies (2.17). By our construction of $K_{n}^{(+)}$it will follow that $K_{n}^{(+)} \rightarrow K^{(+)}$, where $K^{(+)}$is the desired kernel. In general $K^{(+)}(x, t)$ will not satisfy (2.16).

Following Gasymov, for $t \geqslant x$ we define 


$$
A^{(0)}(x, t)=D\left(\frac{x+t}{2}\right)=\left(\begin{array}{cc}
-\frac{1}{2} p((x+t) / 2)+c \int_{(x+t) / 2}^{\infty} v(r) d r & -\frac{1}{2} v((x+t) / 2) \\
-\frac{1}{2} v((x+t) / 2) & \frac{1}{2} p((x+t) / 2)+c \int_{(x+t) / 2}^{\infty} v(r) d r
\end{array}\right)
$$

and then successively $A^{(n+1)}(x, t)=\left(\mathscr{K} A^{(n)}\right)(x, t)$, where $\mathscr{K}$ is the operator on the right-hand side of $(2.17)$ with the term $D(x+t) / 2)$ omitted. One then investigates convergence of the series $A^{(0)}+A^{(1)}+A^{(2)}+\cdots$. We will obtain bounds on the terms $\left|A_{i j}^{(n)}(x, t)\right|$ by induction. The following lemma will give us useful flexibility in working with the operator $\mathscr{K}$.

Lemma 2.2: (i) Let $g(x)$ be a positive function in $L_{1} \cap L_{2}$ that also obeys (1.9). Suppose that $\left|A_{j j}^{(0)}(x, t)\right| \leqslant \sigma_{g}((x+t) / 2)$ and $\left|A_{i j}^{(0)}(x, t)\right|<g((x+t) / 2)(i \neq j)$. Then for $n \geqslant 1$,

$$
\begin{aligned}
& \left|A_{11}^{(n)}(x, t)\right|,\left|A_{22}^{(n)}(x, t)\right| \leqslant\left(c_{n} / n !\right) \sigma_{g}((x+t) / 2)\left[\sigma_{f}^{(1)}(x)\right]^{n} \alpha^{n}(x), \\
& \left|A_{21}^{(n)}(x, t)\right|,\left|A_{12}^{(n)}(x, t)\right| \leqslant\left(c_{n} / n !\right) \sigma_{g}((x+t) / 2)\left[\sigma_{f}^{(1)}(x)\right]^{n}(|x|+1)^{-1} \alpha^{n}(x),
\end{aligned}
$$

where $c_{n}=4^{n}(2 c+1)^{n} c_{0}$ and $c_{0}$ is a constant.

(ii) Suppose that $\left|A_{j}^{(0)}(x, t)\right| \leqslant C$ and $\left|A_{i j}^{(0)}(x, t)\right| \leqslant C(|x|+1)^{-1}(i \neq j)$. Then

$$
\begin{aligned}
& \left|A_{11}^{(n)}(x, t)\right|,\left|A_{22}^{(n)}(x, t)\right| \leqslant\left(d_{n} / n !\right)\left[\sigma_{f}^{(1)}(x)\right]^{n} \alpha^{n}(x), \\
& \left|A_{21}^{(n)}(x, t)\right|,\left|A_{12}^{(n)}(x, t)\right| \leqslant\left(d_{n} / n !\right)\left[\sigma_{f}^{(1)}(x)\right]^{n}(|x|+1)^{-1} \alpha^{n}(x),
\end{aligned}
$$

where $d_{n}=4^{n}(c+1)^{n} d_{0}$ and $d_{0}$ is a constant.

Proof: (i) Beginning with $n=1$ we have first that

$$
\begin{aligned}
A_{11}^{(1)}(x, t)= & c \int_{x}^{(x+t) / 2} v(s) \int_{t+x-s}^{t-x+s} A_{11}^{(0)}(s, u) d u d s-c \int_{x}^{(x+t) / 2} p(s) \int_{t+x-s}^{t-x+s} A_{21}^{(0)}(s, u) d u d s \\
& +c \int_{(x+t) / 2}^{\infty} v(s) \int_{s}^{t-x+s} A_{11}^{(0)}(s, u) d u d s-c \int_{(x+t) / 2}^{\infty} p(s) \int_{s}^{t-x+s} A_{21}^{(0)}(s, u) d u d s \\
& -\frac{1}{2} \int_{x}^{\infty} v(s)\left(A_{21}^{(0)}+A_{12}^{(0)}\right)(s, t-x+s) d s-\frac{1}{2} \int_{x}^{\infty} p(s)\left(A_{22}^{(0)}-A_{11}^{(0)}\right)(s, t-x+s) d s \\
& -\frac{1}{2} \int_{x}^{(x+t) / 2} v(s)\left(A_{21}^{(0)}-A_{12}^{(0)}\right)(s, t+x-s) d s-\frac{1}{2} \int_{x}^{(x+t) / 2} p(s)\left(A_{11}^{(0)}+A_{22}^{(0)}\right)(s, t+x-s) d s \\
= & (1)+(2)+(3)+(4)+(5)+(6)+(7)+(8),
\end{aligned}
$$

where we number the terms on the right-hand side of $(2.20)(1)-(8)$ for reference. We have first that

$$
\begin{aligned}
|(1)| & <c \int_{x}^{(x+t) / 2}|v(s)| \int_{t+x-s}^{t-x+s}\left|A_{11}^{(0)}(s, u)\right| d u d s \leqslant c \int_{x}^{(x+t) / 2}|v(s)| \int_{t+x-s}^{t-x+s}\left(\int_{(u+s) / 2}^{\infty} g(r) d r\right) d u d s \\
& <2 c \int_{x}^{(x+t) / 2}|v(s)| \int_{(x+t) / 2}^{(2 s+t-x) / 2}\left(\int_{r}^{\infty} g(\tau) d \tau\right) d r d s \leqslant 2 c \sigma_{g}\left(\frac{x+t}{2}\right) \int_{x}^{(x+t) / 2}|v(s)|(s-x) d s \\
& <2 c \sigma_{g}\left(\frac{x+t}{2}\right) \sigma_{f}^{(1)}(x) \alpha(x),
\end{aligned}
$$

where we used (1.7), (1.8), and the inequality $s-x \leqslant(|s|+1) \alpha(x)$. For the second term in $(2.20)$,

$$
\begin{aligned}
|(2)| & <\frac{1}{2} c \int_{x}^{(x+t) / 2}|p(s)| \int_{t+x-s}^{t-x+s} g\left(\frac{s+u}{2}\right) d u d s \\
& <c \int_{x}^{(x+t) / 2}|p(s)| \int_{(x+t) / 2}^{(2 s+t-x) / 2} g(r) d r d s \leqslant c \sigma_{g}\left(\frac{x+t}{2}\right) \sigma_{f}^{(1)}(x),
\end{aligned}
$$

also by (1.7) and (1.8). By the same arguments, the third and fourth terms in (2.20) satisfy

$$
\begin{aligned}
|(3)| & <c \int_{(x+t) / 2}^{\infty}|v(s)| \int_{s}^{t-x+s}\left(\int_{(u+s) / 2}^{\infty} g(\tau) d \tau\right) d u d s \leqslant 2 c \sigma_{g}\left(\frac{x+t}{2}\right) \sigma_{f}^{(1)}\left(\frac{x+t}{2}\right) \alpha(x), \\
|(4)| & <c \int_{(x+t) / 2}^{\infty}|p(s)| \int_{s}^{t-x+s} g\left(\frac{s+u}{2}\right) d u d s \leqslant 2 c \int_{(x+t) / 2}^{\infty}|p(s)| \int_{s}^{(2 s+t-x) / 2} g(r) d r d s \\
& <2 c \sigma_{g}\left(\frac{x+t}{2}\right) \sigma_{f}^{(1)}\left(\frac{x+t}{2}\right) .
\end{aligned}
$$

For (3) we used $t-x \leqslant 2(|s|+1) \alpha(x)$.

To estimate term (5) we use (1.9); thus

$|(5)|<\int_{x}^{\infty}|v(s)| g\left(\frac{2 s+t-x}{2}\right) d s \leqslant\left(\int_{x}^{\infty}|v|^{2}\right)^{1 / 2}\left(\int_{(x+t) / 2}^{\infty} g^{2}\right)^{1 / 2} \leqslant \sigma_{f}(x) \sigma_{g}\left(\frac{x+t}{2}\right) \leqslant \sigma_{f}^{(1)}(x) \sigma_{g}\left(\frac{x+t}{2}\right)$. 
For the remaining terms we have (7) $=0$ since $A_{12}^{(0)}=A_{21}^{(0)}$,

$$
|(6)| \leqslant \int_{x}^{\infty}|p(s)| \sigma_{g}\left(\frac{2 s+t-x}{2}\right) d s \leqslant \sigma_{g}\left(\frac{x+t}{2}\right) \sigma_{f}^{(1)}(x)
$$

by (1.8), and

$$
|(8)| \leqslant \int_{x}^{(x+t) / 2}|p(s)| \int_{(x+t) / 2}^{\infty} g(u) d u d s \leqslant \sigma_{g}\left(\frac{x+t}{2}\right) \sigma_{f}^{(1)}(x) .
$$

Adding these terms implies that

$$
\left|A_{11}^{(1)}(x, t)\right| \leqslant C \sigma_{g}((x+t) / 2) \sigma_{f}^{(1)}(x) \alpha(x)
$$

and, similarly,

$$
\left|A_{22}^{(1)}(x, t)\right| \leqslant C \sigma_{g}((x+t) / 2) \sigma_{f}^{(1)}(x) \alpha(x),
$$

where $C$ is a constant and where in the inequalities for (3) and (4) we used $\sigma_{f}^{(1)}((x+t) / 2) \leqslant \sigma_{f}^{(1)}(x)$ since $t \geqslant x$.

As regards $A_{12}^{(n)}(x, t)$, let us begin with

$$
\begin{aligned}
A_{12}^{(1)}(x, t)= & \frac{1}{2} \int_{x}^{\infty} v(s)\left(A_{22}^{(0)}-A_{11}^{(0)}\right)(s, t-x+s)+\frac{1}{2} \int_{x}^{\infty} p(s)\left(A_{21}^{(0)}+A_{12}^{(0)}\right)(s, t-x+s) \\
& +\frac{1}{2} \int_{x}^{(x+t) / 2} v(s)\left(A_{22}^{(0)}+A_{11}^{(0)}\right)(s, t+x-s)-\frac{1}{2} \int_{x}^{(x+t) / 2} p(s)\left(-A_{12}^{(0)}+A_{21}^{(0)}\right)(s, t+x-s) \\
= & (9)+(10)+(11)+(12) .
\end{aligned}
$$

This time (1.7) and (1.8) give

$$
\begin{aligned}
& |(9)| \leqslant \int_{x}^{\infty}|v(s)| \sigma_{g}\left(\frac{2 s+t-x}{2}\right) \leqslant \sigma_{g}\left(\frac{x+t}{2}\right) \sigma_{f}(x), \\
& |(10)| \leqslant \int_{x}^{\infty}|p(s)| g\left(\frac{2 s+t-x}{2}\right) \leqslant \sigma_{g}\left(\frac{x+t}{2}\right) \sigma_{f}(x), \\
& |(11)| \leqslant \int_{x}^{(x+t) / 2}|v(s)| \int_{(t+x) / 2}^{\infty}|g(r)| \leqslant c \sigma_{g}\left(\frac{x+t}{2}\right) \sigma_{f}(x),
\end{aligned}
$$

and $A_{12}^{(0)}=A_{21}^{(0)}$ implies (12) $=0$. Consequently,

$$
\left|A_{12}^{(1)}(x, t)\right| \leqslant C \sigma_{g}((x+t) / 2) \sigma_{f}^{(1)}(x)(|x|+1)^{-1} \alpha(x)
$$

because $\sigma_{f}(x) \leqslant\left[\sigma_{f}^{(1)}(x) /(|x|+1)\right] \alpha(x)$ by an obvious argument. Similarly,

$$
\left|A_{21}^{(1)}(x, t)\right| \leqslant C \sigma_{g}((x+t) / 2) \sigma_{f}^{(1)}(x)(|x|+1)^{-1} \alpha(x),
$$

where $C$ is a constant. We have now verified (2.18) for $n=1$. To obtain $A_{11}^{(n+1)}(x, t)$ we simply replace $A_{j k}^{(0)}$ in (2.18) by $A_{j k}^{(i)}$; this leads to the terms $(1),(2), \ldots,(8)$, which we can estimate as before but now do by using the induction hypothesis (2.18). For the first term,

$$
\begin{aligned}
|(1)| & \leqslant c \int_{x}^{(x+t) / x}|v(s)| \int_{t+x-s}^{t-x+s}\left(\frac{c_{n}}{n !}\right) \sigma_{g}\left(\frac{s+u}{2}\right)\left[\sigma_{f}^{(1)}(s)\right]^{n} \alpha^{n}(s) d u d s \\
& \leqslant 2 c\left(\frac{c_{n}}{n !}\right) \int_{x}^{(x+t) / 2}|v(s)|\left[\sigma_{f}^{(1)}(s)\right]^{n} \alpha^{n}(s) \int_{(t+x) / 2}^{(2 s+t-x) / 2} \sigma_{g}(r) d r d s \\
& \leqslant 2 c\left(\frac{c_{n}}{n !}\right) \sigma_{g}\left(\frac{x+t}{2}\right) \int_{x}^{(x+t) / 2}|v(s)|(s-x)\left[\sigma_{f}^{(1)}(s)\right]^{n} \alpha^{n}(s) d s \\
& \leqslant 2 c\left(\frac{c_{n}}{n !}\right) \sigma_{g}\left(\frac{x+t}{2}\right) \alpha^{n+1}(x) \int_{x}^{\infty}(1+|s|) f(s)\left[\sigma_{f}^{(1)}(s)\right]^{n} d s \\
& =2 c\left(\frac{c_{n}}{(n+1) !}\right) \sigma_{g}\left(\frac{x+t}{2}\right)\left[\sigma_{f}^{(1)}(x)\right]^{n+1} \alpha^{n+1}(x)
\end{aligned}
$$

since $(d / d x) \sigma_{f}^{(1)}(x)=-(1+x) f(x)$ and $s-x \leqslant(|s|+1) \alpha(x)$. Next, we have

$$
\begin{aligned}
|(2)| & \leqslant c \int_{x}^{(x+t) / 2}|p(s)| \int_{t+x-s}^{t-x+s}\left(\frac{c_{n}}{n !}\right) \sigma_{g}\left(\frac{s+u}{2}\right) \frac{\left[\sigma_{f}^{(1)}(s)\right]^{n}}{|s|+1} \alpha^{n}(s) d s \\
& \leqslant c\left(\frac{c_{n}}{n !}\right) \sigma_{g}\left(\frac{x+t}{2}\right) \int_{x}^{(x+t) / 2} \frac{|p(s)|}{|s|+1} 2(s-x)\left[\sigma_{f}^{(1)}(s)\right]^{n} \alpha^{n}(s) d s
\end{aligned}
$$




$$
\begin{aligned}
& <2 c\left(\frac{c_{n}}{n !}\right) \alpha^{n+1}(x) \sigma_{g}\left(\frac{x+t}{2}\right) \int_{x}^{(x+t) / 2} f(s)(1+|s|)\left[\sigma_{f}^{(1)}(s)\right]^{n} d s \\
& <2 c\left(\frac{c_{n}}{(n+1) !}\right) \sigma_{g}\left(\frac{x+t}{2}\right)\left[\sigma_{f}^{(1)}(x)\right]^{n+1} \alpha^{n+1}(x)
\end{aligned}
$$

using the derivative argument and the inequality $s-x \leqslant(|s|+1) \alpha(x)$ again. For the third term,

$$
\begin{aligned}
|(3)| & <c \int_{(x+t) / 2}^{\infty}|v(s)| \int_{s}^{t-x+s}\left(\frac{c_{n}}{n !}\right) \sigma_{s}\left(\frac{s+u}{2}\right)\left[\sigma_{f}^{(1)}(s)\right]^{n} \alpha^{n}(s) d s \\
& <2 c\left(\frac{c_{n}}{n !}\right) \alpha^{n+1}(x) \sigma_{g}\left(\frac{x+t}{2}\right) \int_{(x+t) / 2}^{\infty}|v(s)|(1+|s|)\left[\sigma_{f}^{(1)}(s)\right]^{n} d s \\
& <2 c\left(\frac{c_{n}}{(n+1) !}\right) \sigma_{g}\left(\frac{x+t}{2}\right)\left[\sigma_{f}^{(1)}(x)\right]^{n+1} \alpha^{n+1}(x),
\end{aligned}
$$

where this time we used $t-x \leqslant 2(|s|+1) \alpha(x)$. The fourth term satisfies

$$
\begin{aligned}
|(4)| & <c \int_{(x+t) / 2}^{\infty}|p(s)| \int_{s}^{t-x+s}\left(\frac{c_{n}}{n !}\right) \sigma_{g}\left(\frac{s+u}{2}\right) \frac{\left[\sigma_{f}^{(1)}(s)\right]^{n}}{|s|+1} \alpha^{n}(s) d s \\
& <2 c\left(\frac{c_{n}}{n !}\right) \alpha^{n+1}(x) \sigma_{g}\left(\frac{x+t}{2}\right) \int_{(x+t) / 2}^{\infty}|p(s)|\left[\sigma_{f}^{(1)}(s)\right]^{n} d s \\
& <2 c\left(\frac{c_{n}}{n !}\right) \alpha^{n+1}(x) \sigma_{g}\left(\frac{x+t}{2}\right) \int_{(x+t) / 2}^{\infty}(|s|+1) f(s)\left[\sigma_{f}^{(1)}(s)\right]^{n} d s \\
& <2 c\left(\frac{c_{n}}{(n+1) !}\right) \sigma_{g}\left(\frac{x+t}{2}\right)\left[\sigma_{f}^{(1)}(x)\right]^{n+1} \alpha^{n+1}(x)
\end{aligned}
$$

using $|p(s)| /(|s|+1) \leqslant f(s)$ and $(x+t) / 2 \geqslant x$. The fifth term is handled the same way after noting that

$$
\sigma_{g}((2 s+t-x) / 2) \leqslant \sigma_{g}((x+t) / 2) \text {. }
$$

A factor of 2 is introduced owing to the presence of $A_{11}^{(n)}$ and $A_{22}^{(n)}$. Also, the factor $\alpha(x)$ accounts for the possibility that $x<0$. Thus the bound for $(5)$ is $1 / 2 c$ times the bound for (4). In the sixth term a factor of 2 arises in the same way and we replace $p(s)$ by $(1+|s|) f(s)$, yielding the same bound as for (5). The seventh term bound is $1 / 2 c$ times the fourth term bound because the factor 2 arises from the presence of terms $A_{12}^{(n)}$ and $A_{21}^{(n)}$, and we replace $|v(s)| /(|s|+1)$ by $f(s) /(|s|+1)<(|s|+1) f(s)$ by (1.7). Finally, the bound for $(8)$ is the same as for $(5)-(7)$ because we replace $|p(s)|$ by $(1+|s|) f(s)$. Adding everything we find

$$
\begin{aligned}
\left|A_{11}^{(n+1)}(x, t)\right| \leqslant( & \left(c_{n} /(n+1) !\right) \sigma_{g}((x+t) / 2) \\
& \times\left[\sigma_{f}^{(1)}(x)\right]^{n+1} \alpha^{n+1}(x)[4(2 c+1)]
\end{aligned}
$$

and, noting that $c_{n+1}=4(2 c+1) c_{n}$, we obtain (2.18) for $A_{11}^{(n+1)}$.

The proofs for $A_{22}^{(n+1)}, A_{12}^{(n+1)}$, and $A_{21}^{(n+1)}$ are handled in the same way, and we omit the details. Part (i) of Lemma 2.2 is now proved. Part (ii) follows in a similar way; the details are again omitted.

The following lemma will be needed for the approximation by smooth potentials. It will also enable us to generalize Theorem 2.1 by allowing the coefficient $p(x)$ to be singular as well.
Lemma 2.3: Suppose that $p(x)=v(x)=0$ for $|x|>R$ and that $p(x), v(x) \in L^{2}$. Then for $n \geqslant 1$,

$$
\left|A_{i j}^{(n)}(x, t)\right| \leqslant C \widetilde{C}^{n-1}\left[\sigma_{|v|+|p|}^{n-1}(x) /(n-1) !\right] \alpha^{n}(x),
$$
where $C$ and $\widetilde{C}$ are appropriate constants.

Proof: Note that on the basis of (2.20) we have that $A(x, t)=0$ whenever $x+t>2 R$. Hence the inequality is only of interest in the region $\{(x, t): x<t<2 R-x\}$. First we consider $n=1$. By $(2.20)$ and the subsequent estimates, it follows that $\left|A_{11}^{(1)}(x, t)\right| \leqslant C \alpha(x)$ for some $C$. Note that we may use the Schwarz inequality to estimate term (6) [as we did with (5)] so as to accommodate singularities in $p(x)$. Similar estimates hold for the other matrix elements of $A(x, t)$; for all of them the constant $C$ can be chosen. Considering $n=2$ we conclude from (2.20) that

$$
\left|A_{11}^{(2)}(x, t)\right| \leqslant C \alpha(x)(2 c \alpha(x)+2) \int_{x}^{\infty}(|v(s)|+|p(s)|) d s .
$$

The factor $2 c \alpha(x)+2$ arises from combining terms (1) and $(2),(3)$ and (4), (5) and (6), and (7) and (8). Terms (1)(4) give rise to the extra factor $\alpha(x)$. Hence we put $\widetilde{C}=2 c+2$. The assertion of Lemma 2.3 for $n>2$ now follows by iteration.

Proof of Theorem 2.1: We fix $x_{0}$ arbitrary and assume that $t \geqslant x \geqslant x_{n}$. If $p(x)$ and $v(x)$ satisfy conditions (1.6)(1.9), then $A_{i j}^{(0)}$ satisfies the assumptions of Lemma 2.2 (i) with $g(x)$ being an appropriate multiple of $f(x)$. Then by (2.18), summing $A^{(1)}+A^{(2)}+\cdots$ yields an absolutely and uniformly (on $x_{0} \leqslant x \leqslant t$ ) convergent series such that when the term $A^{(0)}$ is included we obtain a solution of the integral equation (2.17). 
Passing to the next phase of the construction, we will show that with $K^{(+)}=A$ the left-hand side of (2.11) represents the Jost function for (2.9). Now let $R>0$ be arbitrary and define

$$
\begin{aligned}
& p_{R}(x)= \begin{cases}p(x), & x_{0} \leqslant x<R, \\
0, & x>R,\end{cases} \\
& v_{R}(x)= \begin{cases}v(x), & x_{0} \leqslant x<R, \\
0, & x>R,\end{cases}
\end{aligned}
$$

so that $p_{R}$ and $v_{R}$ are cut-off versions of $p$ and $v$. Obviously, (2.17) has a solution corresponding to the cut-off potentials, and we denote it by $A_{R}(x, t)$. The next step is to show

$\int_{x}^{\infty}\left|A(x, t)-A_{R}(x, t)\right| d t \rightarrow 0, \quad R \rightarrow \infty \quad\left(x \geqslant x_{0}\right)$,

Since $A$ and $A_{R}$ satisfy (2.17), then

$$
\begin{aligned}
A-A_{R}= & \left(A^{(0)}-A_{R}^{(0)}\right) \\
& +\left(\mathscr{K}-\mathscr{K}_{R}\right) A_{R}+\mathscr{K}\left(A-A_{R}\right),
\end{aligned}
$$

where $\mathscr{K}_{R}$ is the operator $\mathscr{K}$ with $p$ and $v$ replaced by $p_{R}$ and $v_{R}$, respectively; $A_{R}^{(0)}$ is the corresponding cutoff version of $A^{(0)}$.

If we set

$$
\begin{aligned}
& f_{R}(x)= \begin{cases}f(x), & x>R, \\
0, & x_{0}<x<R,\end{cases} \\
& \sigma_{f_{R}}(x)= \begin{cases}\sigma_{f}(x), & x>R, \\
\sigma_{f}(R), & x_{0}<x<R,\end{cases}
\end{aligned}
$$

and

$$
\sigma_{f_{R}}^{(1)}(x)= \begin{cases}\sigma_{f}^{(1)}(x), & x>R, \\ \sigma_{f}^{(1)}(R), & x_{0}<x<R,\end{cases}
$$

then

$$
\begin{aligned}
& \left|A_{j j}^{(0)}(x, t)-A_{R, i j}^{(0)}(x, t)\right| \leqslant C \sigma_{f_{R}}((x+t) / 2), \\
& \left|A_{i k}^{(0)}(x, t)-A_{R, k}^{(0)}(x, t)\right| \leqslant \frac{1}{2} \cdot f_{R}((x+t) / 2), \quad i \neq k,
\end{aligned}
$$

which accounts for the first term on the right-hand side of (2.22). Looking at the term $\left(\mathscr{K}-\mathscr{K}_{R}\right) A_{R}$ in (2.21), we will use the bounds (2.13) and (2.14) on $A_{R}$ in the form

$$
\begin{aligned}
& \left|A_{R, i i}(x, t)\right| \leqslant C\left(x_{0}\right) \sigma_{f}((x+t) / 2), \\
& \left|A_{R, i k}(x, t)\right| \leqslant C\left(x_{0}\right)[f((x+t) / 2) \\
& \left.\quad+\{1 /(|x|+1)\} \sigma_{f}((x+t) / 2)\right], \quad i \neq k .
\end{aligned}
$$

Here the constant $C\left(x_{0}\right)$ absorbs the dependence on $x_{0}$ that arises from the exponential factors in (2.13) and (2.14). Note that $p_{R}$ and $v_{R}$ obey (1.6)-(1.9) with the same $f$ as $p$ and $v$ do and that $p-p_{R}$ and $v-v_{R}$ obey (1.6)-(1.9) with $f$ replaced by $f_{R}$. Define a matrix $B_{R}(x, t)$ such that $B_{R, i i}(x, t)$ and $B_{R, i k}(x, t)$ are given by the right-hand sides of the inequalities in (2.24), respectively. Then write

$$
B_{R}=B_{R}^{(1)}+B_{R}^{(2)} \text {, }
$$

where

$$
\begin{aligned}
& B_{R, i i}^{(1)}(x, t)=C\left(x_{0}\right) \sigma_{f}((x+t) / 2), \\
& \left.B_{R, i k}^{(1)}(x, t)=C\left(x_{0}\right) f(x+t) / 2\right), \\
& B_{R, i i}^{(2)}(x, t)=0 \\
& B_{R, i k}^{(2)}(x, t)=C\left(x_{0}\right) \sigma_{f}((x+t) / 2)(|x|+1)^{-1} .
\end{aligned}
$$

We can estimate $\left(\mathscr{K}-\mathscr{K}_{R}\right) B_{R}^{(1)}$ by means of part (i) of Lemma 2.2 [with $g=C\left(x_{0}\right) f$ and $f=f_{R}$ ] and $\left(\mathscr{K}-\mathscr{K}_{R}\right) B_{R}^{(2)}$ by means of part (ii). We obtain

$\left|\left(\left(\mathscr{K}-\mathscr{K}_{R}\right) A_{R}(x, t)\right)_{i i}\right| \leqslant C\left(x_{0}\right) \sigma_{f}((x+t) / 2)$

$$
\begin{aligned}
& \times \sigma_{f_{R}}^{(1)}(x) \alpha(x), \\
& =\left(\left(\mathscr{K}_{K}-\mathscr{K}_{R}\right) A_{R}(x, t)\right)_{i k} \mid \leqslant \\
& \left.C\left(x_{0}\right) \sigma_{f}^{(1)}((x)(x) / 25) / 2\right)
\end{aligned}
$$

Putting $D_{R}=A^{(0)}-A_{R}^{(0)}+\left(\mathscr{K}^{\mu}-\mathscr{K}_{R}\right) A_{R}$ and using (2.23) and (2.25) gives

$$
\begin{aligned}
\left|D_{R, i i}(x, t)\right| \leqslant & C\left(x_{0}\right)\left[\sigma_{f_{R}}((x+t) / 2)\right. \\
& \left.+\sigma_{f}((x+t) / 2) \sigma_{f_{R}}^{(1)}(x) \alpha(x)\right], \\
\left|D_{R, i j}(x, t)\right| \leqslant & C\left(x_{0}\right)\left[f_{R}((x+t) / 2)\right. \\
& +\sigma_{f}((x+t) / 2) \sigma_{f_{R}}^{(1)}(x) \\
& \left.\times(|x|+1)^{-1} \alpha(x)\right] .
\end{aligned}
$$

On the basis of $A-A_{R}=D_{R}+\mathscr{K}\left(A-A_{R}\right)$ we can find $A-A_{R}$ by iteration. By Lemma 2.2 , the iterations will produce the factors $C^{n}\left[\alpha(x) \sigma_{f}^{(1)}(x)\right]^{n} / n$ ! that ensure convergence. As a result, we obtain

$$
\begin{aligned}
\left|\left(\left(A-A_{R}\right)(x, t)\right)_{i i}\right| \leqslant & \widetilde{C}\left(x_{0}\right)\left[\sigma_{f_{R}}((x+t) / 2)\right. \\
& \left.+\sigma_{f}((x+t) / 2) \sigma_{f_{R}}^{(1)}(x)\right], \\
\left|\left(\left(A-A_{R}\right)(x, t)\right)_{i k}\right| \leqslant & \widetilde{C}\left(x_{0}\right) \\
& \times\left[f_{R}\left(\frac{x+t}{2}\right)+\sigma_{f_{R}}\left(\frac{x+t}{2}\right)\right. \\
& \left.+\sigma_{f}\left(\frac{x+t}{2}\right) \sigma_{f_{R}}^{(1)}(x)\right] .
\end{aligned}
$$

Now (2.21) follows immediately from these estimates and the fact that $f(x) \in L$ upon letting $R \rightarrow \infty$.

The last stage of the proof will be to replace $A_{R}$ with a kernel arising from smooth cut-off potentials. Thus let $R>0$ continue to be arbitrary and let $\epsilon>0$. One way to approximate the cut-off potentials $p_{R}$ and $v_{R}$ by smooth potentials is by means of the formulas

$$
\begin{aligned}
& p_{R, \epsilon}(x)=\epsilon^{-1} \int_{-\infty}^{\infty} j\left(\frac{t}{\epsilon}\right) p_{R}(x-t) d t, \\
& v_{R, \epsilon}(x)=\epsilon^{-1} \int_{-\infty}^{\infty} j\left(\frac{t}{\epsilon}\right) v_{R}(x-t) d t,
\end{aligned}
$$

where $j(x)$ is a $C^{\infty}$ function with support in, say, $[-1,1]$, and $\int_{-1}^{1} j(x) d x=1$. Then $p_{R, \varepsilon} \rightarrow p_{R}$ and $v_{R, \epsilon} \rightarrow v_{R}, \epsilon \rightarrow 0$, in the $L^{p}$ sense for any $1 \leqslant p<\infty$. Note that $p_{R, \epsilon}$ and $v_{R, \epsilon}$ have support in $[-\epsilon, R+\epsilon]$.

Let $A_{R, \epsilon}(x, t)$ be the solution to (2.17) corresponding to $p_{R, \epsilon}$ and $v_{R, \epsilon}$, i.e.,

$$
A_{R, \epsilon}=A_{R, \epsilon}^{(0)}+\mathscr{K}_{R, \epsilon} A_{R, \epsilon},
$$


where $\mathscr{K}_{R, \epsilon}$ refers to the potential terms $p_{R, \epsilon}$ and $v_{R, \epsilon}$. We will now show that

$\int_{x}^{\infty}\left|A_{R}(x, t)-A_{R, c}(x, t)\right| d t \rightarrow 0, \quad \epsilon \rightarrow 0 \quad\left(x \geqslant x_{0}\right)$.

First we have

$$
\begin{aligned}
A_{R}-A_{R, \epsilon}= & \left(A_{R}^{(0)}-A_{R, \epsilon}^{(0)}\right)+\left(\mathscr{K}_{R}-\mathscr{K}_{R, \epsilon}\right) A_{R, \epsilon} \\
& +\mathscr{K}_{R}\left(A_{R}-A_{R, \epsilon}\right) \\
= & D_{R, \epsilon}+\mathscr{K}_{R}\left(A_{R}-A_{R, \epsilon}\right)
\end{aligned}
$$

using obvious notation for the term $D_{R, \epsilon}$. For fixed $R$ the first term $\left(A_{R}^{(0)}-A_{R, \epsilon}^{(0)}\right) \rightarrow 0, \epsilon \rightarrow 0$ in the $L^{1}$ sense since $p_{R, \epsilon} \rightarrow p_{R}, \quad v_{R, \epsilon} \rightarrow v_{R}$. Next, we consider the term $\left(\mathscr{K}_{R}-\mathscr{K}_{R, \epsilon}\right) A_{R, \epsilon}$. From Lemma 2.3 we know that $\left|A_{R, \epsilon}-A_{R, c}^{(0)}\right| \leqslant C$, where $x+t \leqslant 2 R, x>x_{0}$. This is so because the estimates that lead to the constant $C$ are all given in terms of $L^{1}$ or $L^{2}$ integrals of $v_{R, \epsilon}(x)$ and $p_{R, \epsilon}(x)$ and hence these norms are bounded by constants independent of $\epsilon$ for $\epsilon$ small. Acting with $\left(\mathscr{K}_{R}-\mathscr{K}_{R, \epsilon}\right)$ on $A_{R, \epsilon}$ gives rise to terms that have been discussed in the proof of Lemma 2.2. It follows that

$$
\left|\left(\mathscr{K}_{R}-\mathscr{K}_{R, \epsilon}\right) A_{R, \epsilon}\right| \leqslant C(\epsilon),
$$

where $C(\epsilon) \rightarrow 0$ as $\epsilon \rightarrow 0$. The reason is that the difference $\mathscr{K}_{R}-\mathscr{K}_{R, \epsilon}$ contains the differences $v_{R}-v_{R, \epsilon}$ and $p_{R}-p_{R, \epsilon}$ that appear through their $L^{1}$ and $L^{2}$ norms. Then by iterating (2.20) it follows that (2.21) holds. Note that the $t$ integral in (2.21) is a finite integral since $A_{R}(x, t)$ and $A_{R . \epsilon}(x, t)$ are zero for $x+t \geqslant 2(R+\epsilon)$.

Now go back to the solution $A(x, t)$ of (2.17) and write it as

$$
A=A_{R, \epsilon}+\left(A-A_{R}\right)+\left(A_{R}-A_{R, \epsilon}\right) .
$$

Then $A_{R, \epsilon} \rightarrow A \quad(R \rightarrow \infty, \epsilon \rightarrow 0)$ in the $L^{1}$ sense. The associated potentials $p_{R, \epsilon}$ and $v_{R, \epsilon}$ are smooth and compactly supported. For these potentials, Gasymov's method shows that the PDE (2.16) with $v_{n}=v_{R, \epsilon}$ and $p_{n}=p_{R, \epsilon}$ has a unique solution $K_{R, \epsilon}(x, t)$, which furthermore satisfies (2.17), with the smooth and cut-off potentials and which is such that the function

$$
f_{R, \epsilon}(x, \lambda)=f_{0}(x, \lambda)+\int_{x}^{\infty} K_{R, \epsilon}(x, t) f_{0}(x, \lambda) d t
$$

solves the Dirac equation (2.9) with $v=v_{R, \epsilon}$ and $p=p_{R, \epsilon}$. With $A_{R, \epsilon}=K_{R, \epsilon}$ and introducing $A_{R}$ into (2.30), we can let $R \rightarrow \infty$ and $\epsilon \rightarrow 0$ in (2.31). By the boundedness of $f_{0}(x, \lambda)$, $\lambda \neq \pm c$ and $A_{R, \epsilon} \rightarrow A$ in $L^{1}$, we see that in the limit (2.31) yields

$$
g(x, \lambda)=f_{0}(x, \lambda)+\int_{x}^{\infty} K^{(+)}(x, t) f_{0}(x, \lambda) d t,
$$

where $g$ is defined to be the limit. On the other hand, the function (2.31) is the Jost solution for the Dirac equation with the smooth cut-off potentials, so one has the variation of parameters formula

$$
\begin{aligned}
f_{R, \epsilon}(x, \lambda)= & f_{0}(x, \lambda)-\int_{x}^{\infty} Y_{0}^{-1}(x, \lambda) Y_{0}(t, \lambda) \\
& \times\left(\begin{array}{rr}
-p_{R, \epsilon}(t) & v_{R, \epsilon}(t) \\
v_{R, \epsilon}(t) & p_{R, \epsilon}(t)
\end{array}\right) f_{R, \epsilon}(t, \lambda) d t,
\end{aligned}
$$

where $Y_{0}$ is the fundamental matrix solution of (2.9) with $Y_{0}(0)=I$ and the perturbation terms $p$ and $v$ are dropped. Letting $R \rightarrow \infty$ and $\epsilon \rightarrow 0$ in (2.33) shows that the limit $g(x, \lambda)$ satisfies (1.1); thus $g(x, \lambda)=f(x, \lambda)$ and (2.32) is equivalent to (2.11) with $K=K^{+}$. Since $A(x, t)$ satisfies the bounds (2.13) and (2.14), the proof of Theorem 2.1 is now complete.

The proof for $K^{(-)}$carries over. Note that from (1.6), (1.8), and (1.9) it follows that $|p(x)| \leqslant \int_{-\infty}^{x} f(t) d t$ and that in the estimates integrals of the form $\int^{x}-\infty|h(t)| d t$ and $\int_{-\infty}^{x}(1+|t|)|h(t)| d t$ will replace $\sigma_{h}(x)$ and $\sigma_{h}^{(1)}(x)$, respectively.

Theorem 2.1 can be slightly generalized as follows.

Corollary 2.4: Suppose that conditions (1.6)-(1.9) are satisfied for $|x|>R_{0}$ and that for $|x|<R_{0}$ the coefficients $v(x)$ and $p(x)$ are in $L^{2}$. Then Theorem 2.1 holds.

In other words, the coefficients $p(x)$ and $v(x)$ may be singular inside a bounded region. We only sketch the proof. First, we again write $A-A_{R}=A^{(0)}-A_{R}^{(0)}$ $+\left(\mathscr{K}-\mathscr{K}_{R}\right) A_{R}+\mathscr{K}\left(A-A_{R}\right)$, where $R$ is a cut-off radius such that $R>R_{0}$. In order to control the term $\left(\mathscr{K}-\mathscr{K}_{R}\right) A_{R}$ we write $v_{R}=v_{R, 1}+v_{R, 2}$, where $v_{R, 1}$ is supported in $|x|<R_{0}$ and $v_{R, 2}$ is supported in $R_{0}<|x|<R$. A similar decomposition is introduced for $p_{R}$. Then we have that $A_{R}=A_{R, 1}+A_{R, 2}$, where $A_{R, k}$ solves $A_{R, k}=A_{R, k}^{(0)}+\mathscr{K}_{R} A_{R, k}$. The equation with $k=1$ is now covered by Lemma 2.3 and the equation with $k=2$ is covered by Lemma 2.2. It follows that $A_{R} \rightarrow A$ as $R \rightarrow \infty$ (in the $L^{\prime}$ sense with respect to $t$ ). The matrix $A_{R}$ can be approximated by one involving smooth potentials and the proof of Corollary 2.4 can be completed by following the proof of Theorem 2.1.

For real $\lambda$ such that $f^{( \pm)}(x, \lambda)$ exist, we note that $\left\{f^{(+)}(x, \lambda), \overline{f^{(+)}(x, \lambda)}\right\}$ and $\left\{f^{(-)}(x, \lambda), \overline{f^{(-)}(x, \lambda)}\right\}$ are fundamental sets of solutions to (2.9). Therefore,

$$
f^{(+)}(x, \lambda)=b(\lambda) f^{(-)}(x, \lambda)+a(\lambda) \overline{f^{(-)}(x, \lambda)}
$$

for the scalar functions $a(\lambda)$ and $b(\lambda)$. Using a superscript $T$ to denote transpose and recalling that $\left(y^{T} J z\right)^{\prime}=0$ for two solutions of (2.9) we obtain, from the above equation,

$$
\begin{aligned}
& a(\lambda)=\frac{f^{(-)}(x, \lambda)^{T} J f^{(+)}(x, \lambda)}{\left(f^{(-)}(x, \lambda)^{T} J \bar{f}^{(-)}(x, \lambda)\right.}, \\
& b(\lambda)=\frac{\bar{f}^{(-)}(x, \lambda)^{T} J f^{(+)}(x, \lambda)}{\left({\overline{f^{(-)}(x, \lambda)}}^{T} J f^{(-)}(x, \lambda)\right)}
\end{aligned}
$$

for $\operatorname{Im}(\lambda)>0$. We also introduce

$$
\begin{aligned}
c(\lambda) & =-f^{(-)}(x, \lambda)^{r} J \overline{f^{(-)}(x, \lambda)} \\
& =2(\lambda+c) / i w, \quad \operatorname{Im}(\lambda)>0,
\end{aligned}
$$

where we have put $x=-\infty$ into $f^{(-)}(x, \lambda)^{T} J \overline{f^{(-)}(x, \lambda)}$ and used (2.1). In fact, if we fix $x$ in (2.11), substitute into 
(2.34), and let $|\lambda| \rightarrow \infty$ through real values, the RiemannLebesque theorem shows that

$$
a(\lambda) \rightarrow 1, \quad b(\lambda) \rightarrow 0, \quad|\lambda| \rightarrow \infty, \lambda \text { real. }
$$

It is easy to show that $f^{(+)}(x, \lambda)=O\left(e^{|\operatorname{lm} \lambda| x}\right), x>0$ and $f^{(+)}(x, \lambda)=O\left(e^{-(\operatorname{Im} \lambda) x}\right)$ for $\operatorname{Im} \lambda>0, x \rightarrow+\infty ; \operatorname{sim}-$ ilar results hold for $f^{(-)}(x, \lambda)$. Thus the Phragmén-Lindelöf theorem applies to $a(\lambda)$, so that we further have, from (2.35),

$$
a(\lambda) \rightarrow 1, \quad|\lambda| \rightarrow \infty, \quad \lambda \text { complex. }
$$

As for the scattering matrix on the full line we will need only one entry, namely, the reflection ${ }^{8}$ coefficient defined by

$$
r(\lambda)=b(\lambda) / a(\lambda) .
$$

It follows from (2.36) and (2.37) that $r(\lambda) \rightarrow 0$ as $|\lambda| \rightarrow \infty$ through real values, but it is also true that

$$
r(\lambda)=O(1 / \lambda), \quad|\lambda| \rightarrow \infty .
$$

To prove (2.38) we evaluate the Wronskians in (2.34) and obtain

$$
\begin{aligned}
-r(\lambda)= & \frac{\overline{F^{(-)}(\lambda)}}{F^{(-)}(\lambda)} \cdot \frac{\overline{f_{2}^{(-)}(0, \lambda)}}{f_{1}^{(-)}(0, \lambda)}-\frac{f_{2}^{(+)}(0, \lambda)}{f_{1}^{(+)}(0, \lambda)} \\
& \times\left(\frac{f_{2}^{(-)}(0, \lambda)}{f_{1}^{(-)}(0, \lambda)}-\frac{f_{2}^{(+)}(0, \lambda)}{f_{1}^{(+)}(0, \lambda)}\right)^{-1} \\
= & \frac{\overline{F^{(-)}(\lambda)}}{F^{(-)}(\lambda)} \cdot \frac{\overline{m^{(-)}(\lambda)}-m^{(+)}(\lambda)}{m^{(-)}(\lambda)-m^{(+)}(\lambda)},
\end{aligned}
$$

where $m^{(+)}(\lambda)$ are the Titchmarsh-Weyl coefficients of (2.9) at $\pm \infty$ given by (see Refs, 2, 3, and 5) $m^{( \pm)}(\lambda)=f_{2}^{( \pm)}(0, \lambda) / f_{1}^{( \pm)}(0, \lambda)$. In Ref. 3 one finds that the Jost functions $f_{1}^{( \pm \prime}(0, \lambda)$ are asymptotically constant at $\infty$, and it follows ${ }^{5}$ that $m^{( \pm)}(\lambda)= \pm i+O(1 / \lambda)$, $|\lambda| \rightarrow \infty$. Substituting these estimates into (2.39) yields (2.38).

It is known ${ }^{2}$ that $r(\lambda)$ is continuous in $|\lambda| \geqslant c$ except possibly for $\lambda= \pm c$. For the endpoints we need to make the assumption (compare Frolov ${ }^{8}$ ) that

$r(\lambda)$ is continuous for $|\lambda| \geqslant c$.

If the potentials $v(x)$ and $p(x)$ in (2.9) satisfy the stronger growth condition $v, p \in L_{1}^{1}$, then one can prove ${ }^{28}$ that $r(\lambda)$ is continuous for $|\lambda| \geqslant c$.

We close the section with a corollary to Theorem 2.1 that asserts that the eigenvalues of $(2.9)$ in $(-c, c)$ are finite in number.

Corollary: The number of eigenvalues of (2.9) in $(-c, c)$ is finite.

Proof: We base this proof on the method in Ref. 11. To begin, the eigenvalues coincide with the zeros of the analytic function $a(\lambda)$ in $(-c, c)$. We want to show that these zeros do not cluster in $[-c, c]$. In the vector Hilbert space $L^{2}=\left\{f=\left(f_{1} f_{2}\right)^{T} \mid \int_{-\infty}^{\infty}\left(\left|f_{1}\right|^{2}+\left|f_{2}\right|^{2}\right)<\infty\right\}$ the corresponding eigenfunctions are $f^{(+)}\left(x, \lambda_{j}\right)=b\left(\lambda_{j}\right) f^{(-)}$ $\left(x, \lambda_{j}\right)$, where $\lambda_{j}$ is an eigenvalue. By the orthogonality of eigenfunctions, $\quad \int_{-\infty}^{\infty}\left[f_{1}^{(+)}\left(x, \lambda_{j}\right) f_{1}^{(+)}\left(x, \lambda_{k}\right)\right.$ $\left.+f_{2}^{\{+1}\left(x, \lambda_{j}\right) f_{2}^{\ell+1}\left(x, \lambda_{k}\right)\right] d x=0$; the same relationship holds, of course, for $f^{(-)}\left(x, \lambda_{j}\right)$ and $f^{(-)}\left(x, \lambda_{k}\right)$. By (2.11),

$$
\begin{aligned}
f^{(+)}(x, \lambda)= & \left(\begin{array}{c}
(\lambda+c) / i w \\
1
\end{array}\right) e^{i w x} \\
& +\int_{x}^{\infty} K^{(+)}(x, t)\left(\begin{array}{c}
(\lambda+c) / i w \\
1
\end{array}\right) e^{i w t} d t
\end{aligned}
$$

where $\int_{x}^{\infty}\left|K^{(+)}(x, t)\right| d t \rightarrow 0$ as $x \rightarrow \infty$. For $-c<\lambda<c$ we have that $i w$ is real, and thus both components of $f^{(+)}(x, \lambda)$ are real. Let $w=i \mu$, so that $\lambda=\sqrt{c^{2}-\mu^{2}}$. Since the second term in (2.41) tends to zero, $x \rightarrow \infty$, then for $x_{0}$ sufficiently large,

$$
\begin{aligned}
& f_{i}^{(+)}\left(x, \lambda_{j}\right) f_{i}^{+1}\left(x, \lambda_{k}\right) \\
& \quad \geqslant \frac{\left(c+\sqrt{c^{2}-\mu_{j}^{2}}\right)\left(c+\sqrt{c^{2}-\mu_{k}^{2}}\right)}{4 \mu_{j} \mu_{k}} e^{-\left(\mu_{j}+\mu_{k}\right) x}, \quad x \geqslant x_{0},
\end{aligned}
$$

and thus $f_{1}^{(+)}\left(x, \lambda_{j}\right) f_{\mathrm{i}}^{(+)}\left(x, \lambda_{k}\right)>\left(\left(c+\sqrt{c^{2}-\mu_{0}^{2}}\right)^{2} /\right.$ $\left.4 \mu_{j} \mu_{k}\right) e^{-\left(\mu_{j}+\mu_{k}\right) x}$ for $0<\mu_{j} \leqslant \mu_{0}$ and $0<\mu_{k} \leqslant \mu_{0}$, with $\mu_{0}$ fixed, and $x \geqslant x_{0}$. Similarly, by (2.41),

$$
\begin{aligned}
& f_{2}^{(+)}\left(x, \lambda_{j}\right) f_{2}^{(+)}\left(x, \lambda_{k}\right) \\
& \quad \leqslant e^{-\left(\mu_{j}+\mu_{k}\right) x}\left(2+c / 2 \mu_{j}\right)\left(2+c / 2 \mu_{k}\right) .
\end{aligned}
$$

Now choose $\mu_{0}$ so small that $\left(c+\sqrt{c^{2}-\mu_{0}^{2}}\right) /(2 \mu)>2+(c / 2 \mu)$ for $0<\mu \leqslant \mu_{0}$. Then from the above inequalities we obtain, for some $\epsilon_{0}>0$ and

$$
\begin{aligned}
& 0<\mu_{j}, \mu_{k} \leqslant \mu_{0}, \\
& \int_{x_{0}}^{\infty}\left[f_{1}^{(+)}\left(x, \lambda_{j}\right) f_{1}^{(+)}\left(x, \lambda_{k}\right)\right. \\
& \left.\quad+f_{2}^{(+)}\left(x, \lambda_{j}\right) f_{2}^{(+)}\left(x, \lambda_{k}\right)\right] d x \geqslant \epsilon_{0}>0 .
\end{aligned}
$$

A similar result may be formulated for the integral over $\left(-\infty,-x_{0}\right)$ with respect to $f^{(-)}\left(x, \lambda_{i}\right)$ and $f^{(-)}\left(x, \lambda_{k}\right)$. The argument may now be completed as in Marchenko ${ }^{11}$ (pp. 300-301) by assuming a cluster point $\lambda_{0}(= \pm c)$ of eigenvalues, letting $\lambda_{j}, \lambda_{k} \rightarrow \lambda_{0}$, and then showing that the limit of (2.42) (and its counterpart for $f^{(-)}$) is inconsistent with orthogonality of the eigenfunctions.

\section{THE GLM EQUATION}

We assume (1.6) $-(1.9)$ and (2.40) and begin with the eigenvalue problem (2.9). The spectrum is continuous in $(-\infty,-c]$ and $[c, \infty)$, and there are at most finitely many eigenvalues $\lambda_{1}, \ldots, \lambda_{N}$ in $(-c, c)$. By (2.34) the eigenvalues are the (simple) zeros of $a(\lambda)$ (because $f^{(-)}$and $f^{(+)}$are linearly independent: Note by $(2.11)$ that $f^{( \pm)}(x, \lambda)$ is continuous and real in the gap $-c<\lambda<c$, where $w(\lambda)$ is purely imaginary; thus $a(\lambda)$ is real analytic in the gap). Thus we can form the constants

$$
c_{j}=1 / a^{\prime}\left(\lambda_{j}\right), \quad 1 \leqslant j \leqslant N .
$$

We also let $w_{j}=\sqrt{\lambda_{j}^{2}-c^{2}}$. Recalling the continuity of $r(\lambda)$, define

$$
\begin{aligned}
t_{1}(x)= & \int_{-\infty}^{\infty} r(\lambda)\left(\begin{array}{cc}
-(\lambda+c) / i w & 1 \\
1 & (\lambda-c) / i w
\end{array}\right) \\
& \times\left(\frac{i w}{2 \lambda}\right) e^{-i w x} d w
\end{aligned}
$$


where we integrate with respect to $w(w d w=\lambda d \lambda)$ and view $\lambda=\lambda(w)=\sqrt{w^{2}+c^{2}}$. Finally, let

$$
\begin{aligned}
R(x)= & \frac{\left(t_{1}(x)+\overline{t_{1}(x)}\right)}{2 \pi}+\sum_{j=1}^{N}\left(\frac{c_{j} b\left(\lambda_{j}\right)}{2}\right) e^{-i w_{j} x} \\
& \times\left(\begin{array}{cc}
-\left(\lambda_{j}+c\right) / i w_{j} & 1 \\
1 & \left(\lambda_{j}-c\right) / i w_{j}
\end{array}\right)
\end{aligned}
$$

for all $x$.

Theorem 3.1: The transformation kernel $K^{(-)}(x, t)$ of (2.9) subject to (1.6) $-(1.9)$ satisfies

$$
\begin{aligned}
& R(x+y)+K^{(-)}(x, y) \\
& \quad+\int_{-\infty}^{x} K^{(-)}(x, t) R(t+y) d t=0
\end{aligned}
$$

for arbitrary $x$ and $y, x<y$.

Remark: Theorem 3.1 is the matrix GLM equation for (2.9). A similar equation holds for the kernel $K^{(+)}(x, t)$, with the integral ranging over $x<t<\infty$. To formally solve the inverse problem one starts with $r(\lambda)$, substitutes it into
(3.2), solves (3.3), and obtains the potential in (2.9) via (2.16). The problem of characterizing the scattering data, i.e., giving necessary and sufficient conditions on $r(\lambda)$ such that it should be the reflection coefficient for an operator (2.9) with coefficients in some fixed class, such as that described by (1.6)-(1.9), has been discussed in Ref. 9. Gasymov ${ }^{18}$, who has obtained the necessary and sufficient conditions for the corresponding half-line problem; those conditions, as is true for the Schrödinger case, ${ }^{11}$ are extremely complicated. We do not address the characterization question in this paper, but instead go through the formal inversion procedure (3.2) and (3.3) in Sec. IV with some explicit calculations.

Proof: Following the ideas in Ref. 11 we write

$$
\begin{aligned}
{[1 / a(\lambda)-1] f^{(+)}(x, \lambda)=} & r(\lambda) f^{(-)}(x, \lambda) \\
& +\overline{f^{(-)}(x, \lambda)}-f^{(+)}(x, \lambda)
\end{aligned}
$$

by (2.37) and the definitions of $a(\lambda)$ and $b(\lambda)$. Now substituting from (2.11) we obtain

$$
\begin{aligned}
{\left[\frac{1}{a(\lambda)}-1\right] f^{(+)}(x, \lambda)=} & r(\lambda)\left[e^{-i w x}\left(\begin{array}{c}
-(\lambda+c) / i w \\
1
\end{array}\right)+\int_{-\infty}^{x} K^{(-)}(x, t) e^{-i w t}\left(\begin{array}{c}
-(\lambda+c) / i w \\
1
\end{array}\right) d t\right] \\
& +\left[e^{i w x}\left(\begin{array}{c}
(\lambda+c) / i w \\
1
\end{array}\right)+\int_{-\infty}^{x} K^{(-)}(x, t) e^{i w t}\left(\begin{array}{c}
(\lambda+c) / i w \\
1
\end{array}\right) d t\right] \\
& -\left[e^{i w x}\left(\begin{array}{c}
(\lambda+c) / i w \\
1
\end{array}\right)+\int_{x}^{\infty} K^{(+)}(x, t) e^{i w t}\left(\begin{array}{c}
(\lambda+c) / i w \\
1
\end{array}\right) d t\right] .
\end{aligned}
$$

In (3.4) $x$ is arbitrary and $\lambda$ is a complex number not in the continuous spectrum $-\infty<\lambda \leqslant-c, c \leqslant \lambda<\infty$. Let $y<x$ and multiply (3.4) by $e^{-i \omega y}((\lambda+c) / i w, 1) p(\lambda)$, where

$$
p(\lambda)=\left(\frac{i}{2}\right)\left(\begin{array}{cc}
0 & 1 \\
1 & -2 c / i w
\end{array}\right)
$$

and integrate the result over the counterclockwise contour $C_{R}$ consisting of the circle $|\lambda|=R$, for large $R$, indented with horizontal slits from $-R$ to $-c$ and from $c$ to $R$. The slits should actually be parallel lines slightly above and below the real axis and capped at $\pm c$ by small semicircles, so that the contour avoids the continuous spectrum. We will let $R \rightarrow \infty$ and shrink the contour to horizontal slits. The result of multiplying the left-hand side of (3.4) by $e^{-i \omega y}((\lambda+c) / i w, 1) p(\lambda)$ is real in the gap $-c<\lambda<c$ since $w=w(\lambda)=\sqrt{\lambda^{2}-c^{2}}$ is imaginary there. Hence the new left-hand side continues analytically into the lower halfplane by reflection except for its only singularitics, which are the zeros of $a(\lambda)$ (the eigenvalues) in the gap. Summing the residues gives, for the integral $\left[f^{+}(x, \lambda)\right.$ is analytic $]$,

$$
\begin{aligned}
2 \pi i & \sum_{j=1}^{N} c_{j} f^{(+1}\left(x, \lambda_{j}\right) e^{-i w_{j} y}\left(\frac{\lambda_{j}+c}{i w_{j}}, 1\right) p\left(\lambda_{j}\right) \\
& =2 \pi i \sum_{j=1}^{N} c_{j} b\left(\lambda_{j}\right)\left[e^{-i w_{j} x}\left(\begin{array}{c}
-\left(\lambda_{j}+c\right) / i w_{j} \\
1
\end{array}\right)\right.
\end{aligned}
$$

$$
\begin{aligned}
& \left.+\int_{-\infty}^{x} K^{(-)}(x, t) e^{-i w_{j} t}\left(\begin{array}{c}
-(\lambda+c) / i w_{j} \\
1
\end{array}\right) d t\right] \\
& \times e^{-i w_{j} y}\left(\frac{\lambda_{j}+c}{i w_{j}}, 1\right) p\left(\lambda_{j}\right),
\end{aligned}
$$

where we used $a\left(\lambda_{j}\right)=0$ and, consequently, $f^{(+)}\left(x, \lambda_{j}\right)=b\left(\lambda_{j}\right) f^{(-)}\left(x, \lambda_{j}\right)$ and then (2.11). Eventually, the shrinking/expanding contour encloses all the eigenvalues in $(-c, c)$, so that (3.5) gives the value of the limit, as $R \rightarrow \infty$ and the capped horizontal segments approach slits, of the integral over $C_{R}$. Hence (3.5) also equals the value of the integral of the right-hand side of (3.4), multiplied by $e^{-i w y}((\lambda+c) / i w, 1) p(\lambda)$, over the rays $|\lambda| \geqslant c$, where each ray $\lambda \leqslant-c$ and $\lambda \geqslant c$ is integrated twice. The ray $\lambda \geqslant c$ is integrated forward over the top edge and backward over the bottom edge, and similarly for $\lambda \leqslant-c$. We have used the fact that $a(\lambda) \rightarrow 1,|\lambda| \rightarrow \infty$, and that $f^{(+)}(x, \lambda) e^{-i \omega y}$ is bounded for $y<x$ and $\operatorname{Im}(\hat{\lambda})>0$ by (2.1), so that the contribution from the outer loop $|\lambda|=R$ vanishes as $R \rightarrow \infty$. We also used the fact that $r(\lambda)$ and the factor $e^{-i \omega y}((\lambda+c) / i w, 1) p(\lambda)$ contain only integrable terms near $\lambda= \pm c$, so that the contributions from the small semicircles around $\pm c$ also vanish as the contour shrinks. Finally, the values of the integrand along the top and bottom edges of each ray $|\lambda| \geqslant c$ are complex conjugates of each other.

The right-hand side of (3.4), including the multiplicative factor, is 


$$
\begin{aligned}
& r(\lambda) e^{-i \omega x}\left(\begin{array}{c}
(\lambda+c) /-i w \\
1
\end{array}\right) e^{-i \omega y}\left(\frac{\lambda+c}{i w}, 1\right) p(\lambda)+r(\lambda) \int_{-\infty}^{x} K^{(-)}(x, t) e^{-i \omega t}\left(\begin{array}{c}
(\lambda+c) /-i \omega \\
1
\end{array}\right) d t \\
& \cdot e^{-i \omega y}\left(\frac{\lambda+c}{i \omega}, 1\right) p(\lambda)+\int_{-\infty}^{x} K^{(-)}(x, t) e^{i \omega t}\left(\frac{\lambda+c}{i \omega t}\right) d t \cdot e^{-i \omega y}\left(\frac{\lambda+c}{i \omega}, 1\right) p(\lambda) \\
& -\int_{x}^{\infty} K^{(+)}(x, t) e^{i w t}\left(\begin{array}{c}
\frac{\lambda+c}{i w} \\
1
\end{array}\right) d t \cdot e^{-i w y}\left(\frac{\lambda+c}{i w}, 1\right) p(\lambda) .
\end{aligned}
$$

Let us designate the integrals of the terms in (3.6) by I-IV and evaluate each in turn. For the first term we have $\mathrm{I}=t_{1}+\bar{t}_{1}$, where $t_{1}=t_{1}(x+y)$ as in $(3.1)$ :

$$
\begin{aligned}
t_{1}= & \int_{|\lambda|>c} r(\lambda) e^{-i w x}\left(\begin{array}{c}
-(\lambda+c) / i w \\
1
\end{array}\right) \\
& \times e^{-i \omega y}((\lambda+c) / i w, 1) p(\lambda) d \lambda \\
= & \left(\frac{i}{2}\right) \int_{-\infty}^{\infty} r(\lambda)\left(\begin{array}{cc}
(\lambda+c)^{2} / w^{2} & (\lambda+c) /-i w) \\
(\lambda+c) / i w & 1
\end{array}\right) \\
& \times\left(\begin{array}{cc}
0 & 1 \\
1 & -2 c / i w
\end{array}\right) e^{-i w(x+y)} \frac{w(\lambda) d w}{\lambda} \\
= & \int_{-\infty}^{\infty} r(\lambda)\left(\begin{array}{cc}
(\lambda+c) / i w & 1 \\
1 & (\lambda-c) / i w
\end{array}\right) \\
& \times\left(\frac{i w}{2 \lambda}\right) e^{-i w(x+y)} d w,
\end{aligned}
$$

where we used

$$
\frac{(\lambda+c)^{2}}{w^{2}}-\frac{2 c(\lambda+c)}{w^{2}}=\frac{(\lambda+c)(\lambda-c)}{w^{2}}=1
$$

and $\lambda d \lambda=w d w$. The exponential factor coupled with (2.38) makes the integral converge. The second term is given by II $=t_{2}+\bar{t}_{2}$, where noting the bounds $(2.13)$ and (2.14) (for $\left.K^{(-)}\right)$,

$$
\begin{aligned}
t_{2}= & \int_{|\lambda|>c} r(\lambda) \int_{-\infty}^{x} K^{(-)}(x, t) e^{-i w t}\left(\begin{array}{c}
-(\lambda+c) / i w \\
1
\end{array}\right) \\
& \times d t \cdot e^{-i w y}((\lambda+c) / i w, 1) p(\lambda) d \lambda \\
= & \int_{-\infty}^{\infty} r(\lambda) \int_{-\infty}^{x} K^{(-)}(x, t) \\
& \times\left(\begin{array}{cc}
(\lambda+c) /-i w & 1 \\
1 & (\lambda-c) / i w
\end{array}\right) \\
& \times\left(\frac{i w}{2 \lambda}\right) e^{-i w(t+y)} d t d w \\
= & \int_{-\infty}^{x} K^{(-)}(x, t) t_{1}(y+t) d t
\end{aligned}
$$

after interchanging the order of integration that will now be justified. It will be sufficient to treat one component of (3.7) at a time, and we look only at

$$
\begin{aligned}
& \int_{-\infty}^{\infty} r(\lambda) \int_{-\infty}^{x}\left(K_{11}^{(-)}(x, t)\left(\frac{\lambda+c}{-i w}\right)+K_{12}^{(-)}(x, t)\right) \\
& \times\left(\frac{i w}{2 \lambda}\right) e^{-i w(t+y)} d t d w .
\end{aligned}
$$

It is further sufficient to justify interchanging the order of integration for $\int_{-\infty}^{\infty} r(\lambda) \int_{-\infty}^{x} k(x, t) e^{-i w(t+y)} d t d w$, where $k(x, t)$ is a scalar function satisfying (2.13) and (2.14). This expression is the limit as $M \rightarrow \infty$ of

$$
\begin{array}{rl}
\int_{-M}^{M} & r(\lambda) \int_{-\infty}^{x} k(x, t) e^{-i w(t+y)} d t d w \\
& =\int_{-\infty}^{x} k(x, t) \int_{-M}^{M} r(\lambda) e^{-i w(t+y)} d w d t \\
& =\int_{-\infty}^{x} k(x, t) G_{M}(t+y) d t
\end{array}
$$

with obvious notation, and $G_{M}(t+y)$ has a limit $G(t+y)$ as $M \rightarrow \infty$ by (2.38) and the exponential factor. By the Plancherel theorem ${ }^{29} \quad G_{M} \rightarrow G$ in $L^{2}$ and $G \in L^{2}$, so that $\left\|k(x,)\left(G_{M}-G\right)\right\|_{1} \leqslant\|k(x,)\|_{2}\left\|G_{M}-G\right\|_{2} \rightarrow 0, \quad M \rightarrow \infty$, i.e., $\quad \int_{-\infty}^{x} k(x, t) G_{M}(t+y) d t \rightarrow \int_{-\infty}^{x} k(x, t) G(t+y) d t$. Therefore, letting $M \rightarrow \infty$ in (3.8) justifies the interchange and proves (3.7).

Passing on to term III in (3.6) we have III $=t_{3}+\bar{t}_{3}$ with

$$
\begin{aligned}
t_{3}= & \int_{|\lambda|>c}\left(\int_{-\infty}^{x} K^{(-)}(x, t) e^{i w(t-\mu)} d t\right)\left(\frac{i}{2}\right) \\
& \times\left(\begin{array}{cc}
(\lambda+c) / i w & -1 \\
1 & (\lambda-c) / i w
\end{array}\right) d \lambda .
\end{aligned}
$$

Here we will substitute $\lambda= \pm \sqrt{w^{2}+c^{2}}$, taking the plus sign for $\lambda>c(w>0)$ and the minus sign for $\lambda<-c(w<0)$. Now introduce $T(w)=\int^{x}{ }_{-\infty} K^{(-)}(x, t) e^{i w(t-y)} d t$ and let $s=\sqrt{w^{2}+c^{2}}$ be the positive square root. Then we have

$$
\begin{aligned}
t_{3}= & \int_{-\infty}^{0} T(w)\left(\frac{i}{2}\right)\left(\begin{array}{cc}
(-s+c) / i w & -1 \\
1 & (-s-c) / i w
\end{array}\right) \\
& \times\left(-\frac{w}{s}\right) d w+\int_{0}^{\infty} T(w)\left(\frac{i}{2}\right) \\
& \times\left(\begin{array}{cc}
(s+c) / i w & -1 \\
1 & (s-c) / i w
\end{array}\right)\left(\frac{w}{s}\right) d w
\end{aligned}
$$

where we replaced $\lambda$ by $s$ for $\lambda>c$ and $\lambda$ by $-s$ for $\lambda<-c$. Therefore, 


$$
\begin{aligned}
t_{3}+\bar{t}_{3}= & \int_{-\infty}^{0} T(w)\left(\frac{i}{2}\right)\left(\begin{array}{cc}
-s+c) / i w & -1 \\
1 & (-s-c) / i w
\end{array}\right)\left(-\frac{w}{s}\right) d w \\
& +\int_{0}^{\infty} T(w)\left(\frac{i}{2}\right)\left(\begin{array}{cc}
(s+c) / i w & -1 \\
-1 & (s-c) / i w
\end{array}\right)\left(\frac{w}{s}\right) d w \\
& +\int_{-\infty}^{0} T(-w)\left(-\frac{i}{2}\right)\left(\begin{array}{cc}
(-s+c) /-i w & -1 \\
1 & (-s-c) /-i w
\end{array}\right)\left(-\frac{w}{s}\right) d w \\
& +\int_{0}^{\infty} T(-w)\left(-\frac{i}{2}\right)\left(\begin{array}{cc}
(s+c) /-i w & -1 \\
1 & (s-c) /-i w
\end{array}\right)\left(\frac{w}{s}\right) d w .
\end{aligned}
$$

Replace $w$ by $-w$ in the fourth integral and combine the result with the first, and do the same with the second and third. The result is

$$
\begin{aligned}
t_{3}+\bar{t}_{3}= & \int_{-\infty}^{0} T(w)\left(\frac{i}{2}\right)\left[\left(\begin{array}{cc}
(-s+c) / i w & -1 \\
1 & (-s-c) / i w
\end{array}\right)\left(-\frac{w}{s}\right)+\left(\begin{array}{cc}
(s+c) / i w & -1 \\
1 & (s-c) / i w
\end{array}\right)\left(\frac{w}{s}\right)\right] d w \\
& +\int_{0}^{\infty} T(w)\left(\frac{i}{2}\right)\left[\left(\begin{array}{cc}
(s+c) / i w & -1 \\
1 & (s-c) / i w
\end{array}\right)\left(\frac{w}{s}\right)+\left(\begin{array}{cc}
(-s+c) / i w & -1 \\
1 & (-s-c) / i w
\end{array}\right)\left(-\frac{w}{s}\right)\right] d w \\
= & \int_{-\infty}^{0} T(w)\left(\frac{i}{2}\right)\left(\begin{array}{cc}
2 / i & 0 \\
0 & 2 / i
\end{array}\right) d w+\int_{0}^{\infty} T(w)\left(\frac{i}{2}\right)\left(\begin{array}{cc}
2 / i & 0 \\
0 & 2 / i
\end{array}\right) d w \\
= & \int_{-\infty}^{\infty} T(w) d w=\int_{-\infty}^{\infty} \int_{-\infty}^{\infty} K^{(-)}(x, t) e^{i w(t-y)} d t d w=2 \pi K^{(-)}(x, y)
\end{aligned}
$$

by the inverse Fourier transform and noting that $K^{(-)}(x, t)=0$ for $t>x$. Similar calculations for term IV in (3.6) result in IV $=t_{4}+\bar{t}_{4}$ with $t_{4}+\bar{t}_{4}=2 \pi K^{(+)}(x, y)=0$ since $y<x$.

Equating (3.5) with terms I-IV, we have

$$
\begin{gathered}
2 \pi i \sum_{j=1}^{N} c_{j} b\left(\lambda_{j}\right)\left[e^{-i w_{j}(x+y)} I+\int_{-\infty}^{x} K^{(-)}(x, t) e^{-i w_{j}(t+y)} d t\right]\left(\frac{i}{2}\right)\left(\begin{array}{cc}
\left(\lambda_{j}+c\right) /-i w_{j} & 1 \\
1 & \left(\lambda_{j}-c\right) / i w_{j}
\end{array}\right) \\
\quad=t_{1}(x+y)+\overline{t_{1}(x+y)}+\int_{-\infty}^{x} K^{(-)}(x, t)\left[t_{1}(y+t)+\overline{t_{1}(y+t)}\right] d t+2 \pi K^{(-)}(x, y)
\end{gathered}
$$

and then recalling (3.2) we obtain (3.3). This completes the proof of Theorem 3.1.

\section{RATIONAL REFLECTION COEFFICIENT}

In this section we obtain some explicit solutions to the GLM equation generated by a rational reflection coefficient $r(\lambda)$. We will consider the one- and two-pole cases.

For the one-pole case let $-c<a<0$ and $r(\lambda)$ $=-i a /(w-i a)$. The normalization $r(0)=1$ corresponds to there being no "zero-energy bound state." First let $x<0$. For the function $t_{1}$ of (3.1) we have

$$
\begin{aligned}
t_{1}(x)= & \left(-\frac{i a}{2}\right) \int_{-\infty}^{\infty} \frac{e^{-i w x}}{w-i a}\left[\left(\begin{array}{cc}
-1 & 0 \\
0 & 1
\end{array}\right)\right. \\
& \left.+\frac{c}{\lambda}\left(\begin{array}{cc}
-1 & 0 \\
0 & -1
\end{array}\right)+\frac{i w}{\lambda}\left(\begin{array}{ll}
0 & 1 \\
1 & 0
\end{array}\right)\right] d w \\
= & f_{1}(x)+f_{2}(x)+f_{3}(x),
\end{aligned}
$$

with the obvious notation. We do the integration in (4.1) by the residue theorem using the semicircle $|w|=R$ and the segment $-R \leqslant w \leqslant R$ together with a vertical slit from $w=0$ to $w=i c$; see Fig. 1 .

In actuality, the horizontal path is just above the real $w$ axis and the vertical slit consists of two parallel segments on either side of the imaginary axis and capped with a small semicircle about $i c$; then we let $R \rightarrow \infty$ and shrink the contour. Owing to the term $e^{-i w x}$, which is small on the outer loop since $x<0$, the contribution from the outer loop tends to zero as $R \rightarrow \infty$ by Jordan's lemma. The integral around the full contour vanishes because all terms in (4.1) are analytic inside since $a<0$. Thus (4.1) equals the negative of the inte-

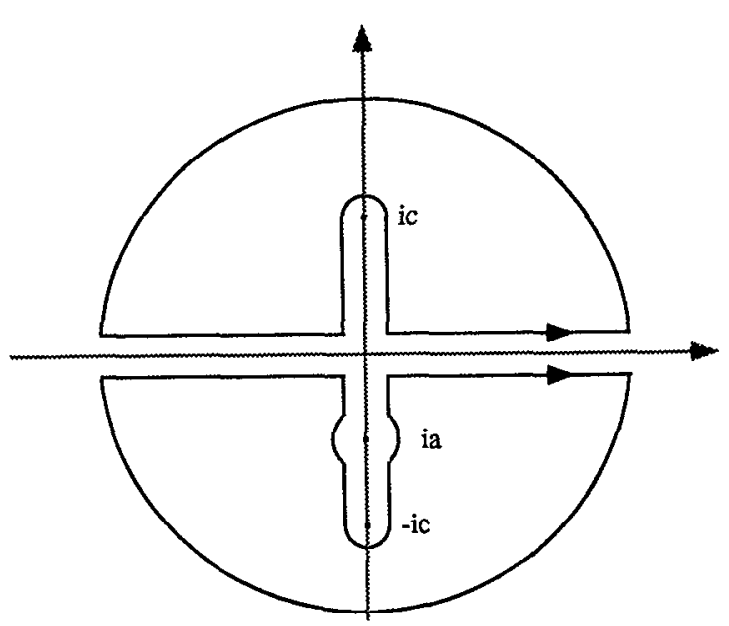

FIG. 1. The integration in Eq. (4.1) by the residue theorem. 
gral over the slit, up the left side and down the right. The integral up and down slit is not zero because $\lambda$ changes signs, negative to positive, crossing over the slit. However, noting (4.1) we see that each $f_{k}(x)$ is purely imaginary since $w$ and $d w$ are purely imaginary on the slit. Checking with (3.2) it follows that $t_{1}(x)$ for $x<0$ makes no contribution to $R(x)$.

In the program of Ref. 14 for the Schrödinger operator the reflection coefficient is rational, with poles on the positive imaginary axis. The function that corresponds to $t_{1}(x)$ is evaluated by summing residues, so that it becomes a sum of exponentials. The same exponentials are fed into the discrete part of (3.2) and the norming constants $c_{j}$ are adjusted so as to cause $R(x)$ to vanish identically for $x<0$; this is crucial for the further evaluation of $R(x)$ for $x>0$ (see our calculation below). Thus in the present case we need to omit the discrete part of (3.2) in order to have $R(x)=0, x<0$. Thus the Dirac system (2.9) is in this context inherently different from the Schrödinger equation.

Thus suppose we omit the discrete spectrum in (3.2), so that $R(x)=0$ for $x<0$, and proceed to calculate $R(x)$ for $x>0$. Form in the lower half-plane the mirror image of the contour used in the $x<0$ case except for the portion of a small circle about $w=i a$, traversed counterclockwise and connecting the nearby vertical paths that will become the slit from zero to - ic when the contour shrinks; see Fig. 1. The radius of the small circle will approach zero as the contour shrinks. As in the former case the contribution to $t_{1}(x)$ from the vertical slit is purely imaginary, so that its contribution to $R(x)$ vanishes. As the contour shrinks the contribution from the small circle about $i a$ has a limit that may be evaluated by integrating around the full (small) circle. If we decompose the integrand as in (4.1) one may calculate that the contributions from the $(c / \lambda)$ and $(i w / \lambda)$ terms are purely imaginary. The residue from the remaining term is

$$
\left(\frac{i a}{2}\right) e^{a x}\left(\begin{array}{cc}
-1 & 0 \\
0 & 1
\end{array}\right)
$$

and, therefore,

$$
\begin{aligned}
& t_{1}(x)=\left(\frac{i a}{2}\right)(2 \pi i) e^{a x}\left(\begin{array}{cc}
-1 & 0 \\
0 & 1
\end{array}\right)=-\pi a e^{a x}\left(\begin{array}{cc}
-1 & 0 \\
0 & 1
\end{array}\right), \\
& R(x)=e^{a x}\left(\begin{array}{cc}
a & 0 \\
0 & -a
\end{array}\right), \quad x>0 .
\end{aligned}
$$

Turning now to the Marchenko equation (3.3) we can first put $x<0$ and $y=x$, so that the terms $R(x+y)=R(2 x)=0$ and $R(t+y)=R(t+x)=0$. Thus the solution is $K^{(-)}(x, x)=0$ and it follows from (2.12) that $p(x)=0$ and $v(x)=0$ for $x<0$ in (2.9). For $x+y>0(3.3)$ becomes

$$
\begin{aligned}
& e^{a(x+y)}\left(\begin{array}{cc}
a & 0 \\
0 & -a
\end{array}\right)+K^{(-)}(x, y) \\
& \quad+\int_{-y}^{x} K^{(-)}(x, t)\left(\begin{array}{cc}
a & 0 \\
0 & -a
\end{array}\right) e^{a(t+y)} d t=0, \\
& \left(\begin{array}{cc}
a & 0 \\
0 & -a
\end{array}\right) e^{a x}+F(y)+\int_{-y}^{x} F(t)\left(\begin{array}{cc}
a & 0 \\
0 & -a
\end{array}\right) e^{2 a t} d t=0,
\end{aligned}
$$

where $F(y)=K^{(-)}(x, y) e^{-a y}$ with $x>0$ fixed. It is the simplified lower limit $-y$ in (4.2), a result of $R(x)=0$ for $x<0$, that allows us to solve (4.2). Considering the $(2,2)$ component of (4.2) the equivalent equation is $F_{22}^{\prime}(y)-a F_{22}(-y) e^{2 a y}=0$. Substituting this into its own derivative gives $F_{22}^{\prime \prime}(y)+2 a F_{22}^{\prime}(y)+a^{2} F_{22}(y)=0$, and we have the boundary conditions $F_{22}(-x)=a e^{\alpha x}$, $F_{22}^{\prime}(-x)=a^{2} e^{a x}$. The general solution to the second-order equation is $F_{22}(y)=c_{1}(x) e^{-a y}+c_{z}(x) y e^{-a y}$, and the boundary conditions imply $c_{1}(x)=a /(1-2 a x)$ and $c_{2}(x)=2 a^{2} /(1-2 a x)$. Thus $F_{22}(y)=a e^{-a y}(1+2 a y) /$ $(1-2 a x)$, and then $K_{22}(x, y)=a(1+2 a y) /(1-2 a x)$. The calculation for $F_{11}$ is similar and leads to $K_{11}^{-\gamma}(x, y)=-a$ for all $x$ and $y$. For the off-diagonal components of $F$ we have the integral equations $F_{i j}(y)$ $\pm a \int_{-y}^{x} F_{i j}(t) e^{2 u t} d t=0$, from which $F_{i j}(x)=0$ follows, and, therefore, $K_{12}^{(-)}(x, y)=K_{21}^{(-)}(x, y)=0$. Finally, (2.16) gives the solutions

$$
v(x)=0, \quad p(x)=-2 a /(1-2 a x), \quad x>0 .
$$

As it happens, we can reverse the analysis in this example by starting with the computed potential $v(x)=p(x)=0 \quad$ for $\quad x<0$ and $v(x)=0$, $p(x)=-2 a /(1-2 a x)$ for $x>0$ and calculate in the "forward" direction that $r(\lambda)=-i a /(w-i a)$. Thus we are able to close the loop starting with the scattering data, going to the potential terms, and then going back to the scattering data. In fact, if for $x>0, v=0$ and $p=-2 a /(1-2 a x)$, then (1.1) becomes $y_{1}^{\prime}=p y_{1}+(\lambda+c) y_{2},-y_{2}^{\prime}$ $=(\lambda+c) y_{1}+p y_{2}$. Thus $y_{1}^{\prime \prime}=p^{\prime} y_{1}+p y_{1}^{\prime}+(\lambda+c)$ $\times\left[-(\lambda-c) y_{1}-p y_{2}\right]=p^{\prime} y_{1}+p y_{1}^{\prime}-w^{2} y_{1}-(\lambda+c) p y_{2}$ $=p^{\prime} y_{1}+p y_{1}^{\prime}-w^{2} y_{1}-p y_{1}^{\prime}+p^{2} y_{1}$. Using the fact that $p^{\prime}=-p^{2}$ this becomes $y_{1}^{\prime \prime}=-w^{2} y_{1}$, so that $y_{1}=c_{1} e^{i \omega x}+c_{2} e^{-i \omega x}$ is the first component of the general solution. Using the above equations one can show that the independent solutions are

$$
\begin{aligned}
& \left(\begin{array}{c}
e^{i \omega x} \\
e^{i \omega x}(i w+2 a /(1-2 a x)) /(\lambda+c)
\end{array}\right), \\
& \left(\begin{array}{c}
e^{-i \omega x} \\
e^{-i \omega x}(-i w+2 a /(1-2 a x)) /(\lambda+c)
\end{array}\right) .
\end{aligned}
$$

Thus for $x>0$, apart from a factor depending only on $\lambda$ and $w$, we have

$$
f^{(+)}(x, \lambda)=e^{i \omega x}\left(\begin{array}{c}
1 \\
(i w+2 a /(1-2 a x)) /(\lambda+c)
\end{array}\right) .
$$

For $f^{(-)}(x, \lambda)$ we match at $x=0$ the values of

$$
f^{(-)}(x, \lambda)=e^{-i \omega x}\left(\begin{array}{c}
1 \\
-i w /(\lambda+c)
\end{array}\right), \quad(x<0)
$$

and

$$
\begin{aligned}
f^{(-1}(x, \lambda) & \\
= & c_{1} e^{i w x}\left(\begin{array}{c}
1 \\
(i w+2 a /(1-2 a x)) /(\lambda+c)
\end{array}\right)+c_{2} e^{-i w x} \\
& \times\left(\begin{array}{c}
1 \\
(-i w+2 a /(1-2 a x)) /(\lambda+c)
\end{array}\right), \quad x>0,
\end{aligned}
$$

to obtain for $x>0$ that 


$$
\begin{aligned}
f^{(-)}(x)= & \left(\frac{i a}{w}\right) e^{i \omega x}\left(\begin{array}{c}
1 \\
(i a+2 a /(1-2 a x)) /(\lambda+c)
\end{array}\right) \\
& +\left(\frac{w-i a}{w}\right) e^{-i w x} \\
& \times\left(\begin{array}{c}
1 \\
(-i w+2 a /(1-2 a x)) /(\lambda+c)
\end{array}\right) .
\end{aligned}
$$

By definition of $r(\lambda), \quad(1 / a(\lambda)) f^{(+)}(x, \lambda)$ $=r(\lambda) f^{(-)}(x, \lambda)-\overline{f^{(-)}(x, \lambda)}$, that is, $r(\lambda)$ must exterminate the exponentially growing terms in $r f^{(-)}-\bar{f}^{(-)}$, and from the above expressions we easily obtain $[r(\lambda)(w-i a)-(-i a)] / w=0$, or $\quad r(\lambda)=-i a /$ $(w-i a)$ as in the beginning.

It is worth noting that for Dirac systems, single-pole reflection coefficients result in finite, discontinuous potentials. For the Schrödinger case, the corresponding case leads to a $\delta$-function potential.

In the degenerate case $c=0$ the slit contours described above are unnecessary; in fact, the theory proceeds much as in Ref. 14. Some examples with rational reflection coefficients are given in Bava et al..$^{24,25}$

Last, we turn to a two-pole case and work through an explicit solution when $r(\lambda)=a b /(w-i a)(w-i b)$ with $-c<a<0$ and $-c<b<0$. Let $r(\lambda)=-a b /$ $(w-i a)(w-i b),-c<a<0$, and $-c<b<0$. According to $(3.1)$,

$$
\begin{aligned}
t_{1}(x)= & \left(-\frac{a b}{2}\right) \int_{-\infty}^{\infty} \frac{e^{-i w x}}{(w-i a)(w-i b)} \\
& \times\left[\left(\begin{array}{cc}
-1 & 0 \\
0 & 1
\end{array}\right)\right. \\
& \left.+\frac{c}{\lambda}\left(\begin{array}{cc}
-1 & 0 \\
0 & -1
\end{array}\right)+\frac{i w}{\lambda}\left(\begin{array}{cc}
0 & 1 \\
1 & 0
\end{array}\right)\right] d w \\
= & f_{1}(x)+f_{2}(x)+f_{3}(x),
\end{aligned}
$$

with obvious notation. For $x<0$ we integrate over the same contour as in the one-pole case for $x<0$ with the result that $t_{1}(x)$ is purely imaginary. Again we omit the discrete part of (3.2), so that $R(x)=0$ for $x<0$ by (3.2).

Now consider $x>0$. This time we evaluate (4.3) by integrating over a contour in the lower half-plane consisting of the real $w$ axis from $-R$ to $R$, where $R>0$, and two vertical lines on either side of and near to the imaginary axis down to $w=-i c$ and capped there with a small semicircle, together with small semicircles about each of $w=i a$ and $w=i b$ as in the previous case. The integral over the whole curve vanishes with its analytic integrand. Integrands over the vertical portions are purely imaginary for $f_{2}(x)$ and $f_{3}(x)$ and cancel for $f_{1}(x)$. Integrals over the circular "bubbles" are purely imaginary for $f_{2}(x)$ and $f_{3}(x)$. Therefore, the only contribution to $R(x)$ comes from the integrals over the bubbles from $f_{1}(x)$. Shrinking the contour and using the residue theorem for the two bubbles $(B)$ we obtain

$$
t_{1}(x)=\left(\frac{a b}{2}\right) \int_{B} \frac{e^{-i w x}}{(w-i a)(w-i b)}\left(\begin{array}{cc}
-1 & 0 \\
0 & 1
\end{array}\right) d w
$$

+ purely imaginary

$$
=(\pi i a b \operatorname{res}(i a)+\pi i a b \operatorname{res}(i b))\left(\begin{array}{cc}
-1 & 0 \\
0 & 1
\end{array}\right)
$$

+ purely imaginary

$$
=(\pi i a b)\left(\begin{array}{cc}
-1 & 0 \\
0 & 1
\end{array}\right)\left(\frac{e^{a x}}{i a-i b}+\frac{e^{b x}}{i b-i a}\right)
$$

+ purely imaginary,

$$
R(x)=\frac{a b}{b-a}\left(e^{b x}-e^{a x}\right)\left(\begin{array}{cc}
-1 & 0 \\
0 & 1
\end{array}\right)
$$

Write this as

$$
R(x)=N\left(e^{b x}-e^{a x}\right), \quad N=\left(\begin{array}{cc}
-1 & 0 \\
0 & 1
\end{array}\right) \frac{a b}{(b-a)} .
$$

As before, we consult (3.3) and put $R(x+t)=0$ for $x+t<0$ to conclude $K^{(-)}(x, t)=0$ for $x+t<0$, so that

$$
\begin{aligned}
& N\left(e^{b(x+y)}-e^{a(x+y)}\right)+K^{(-)}(x, y) \\
& \quad+\int_{-y}^{x} K^{(-)}(x, t) N\left(e^{b(t+y)}-e^{a(t+y)}\right) d t=0 .
\end{aligned}
$$

We proceed to calculate $K^{(-)}(x, y)$ by entries.

For the $(1,1)$ entry let $f(y)=K_{11}^{(-)}(x, y)$, $C=a b /(b-a), x$ fixed. Then

$$
\begin{aligned}
0= & C\left(e^{b(x+y)}-e^{a(x+y)}\right)+f(y) \\
& +\int_{-y}^{x} f(t) C\left(e^{b(t+y)}-e^{a(t+y)}\right) d t .
\end{aligned}
$$

Define and then calculate

$$
\begin{aligned}
I(y)= & \int_{-y}^{x} f(t) C\left(e^{b(t+y)}-e^{a(t+y)}\right) d t, \\
I^{\prime}(y)= & \int_{-y}^{x} f(t) C\left(b e^{b(t+y)}-a e^{a(t+y)}\right) d t, \\
I^{\prime \prime}(y)= & f(-y) C(b-a) \\
& \quad+\int_{-y}^{x} f(t) C\left(b^{2} e^{b(t+y)}-a^{2} e^{a(t+y)}\right) d t .
\end{aligned}
$$

Using this we follow Jordan ${ }^{14}$ and apply the operator $L=(d / d t-b)(d / d t-a)=d^{2} / d t^{2}-(a+b) d / d t$ $+a b$ to (4.5) to obtain

$f^{\prime \prime}(y)-(a+b) f^{\prime}(y)+a b f(y)+a b f(-y)=0$,

to which we attach the boundary conditions $f(-x)=0$ and, from the first derivative of $(4.5), f^{\prime}(-x)=-a b$. Now letting

$$
\begin{aligned}
& \gamma=\left(a^{2}+b^{2}\right)^{1 / 2}, \\
& f_{1}(y)=\cosh \gamma y+[(a+b) / \gamma] \sinh \gamma y, \\
& f_{2}(y)=(a+b) / 2 a b+y,
\end{aligned}
$$

we see that $f_{1}$ and $f_{2}$ are solutions of (4.6). Writing the general solution $f(y)=c_{1} f_{1}(y)+c_{2} f_{2}(y)$ and applying the boundary conditions yields 


$$
\begin{aligned}
c_{1}(x)= & -a b(\cosh \gamma x \\
& -[(a+b) / \gamma] \sinh \gamma x) / W(x), \\
c_{2}(x)= & -a b(x-(a+b) / a b) / W(x), \\
W(x)= & \cosh \gamma x-[(a+b) / \gamma] \sinh \gamma x+\gamma(x-(a \\
& +b) / \gamma)(-\sinh \gamma x+[(a+b) / \gamma] \cosh \gamma x) .
\end{aligned}
$$

One can show that $W(x)$ does not vanish. Feeding these into $f(y)=K_{11}^{-1}(x, y)$ results in

$$
\begin{aligned}
K_{11}^{(-)}(x, y)= & -a b\left[\left(\cosh \gamma x-\frac{a+b}{\gamma} \sinh \gamma x\right)\right. \\
& \times\left(\frac{a+b}{2 a b}+y\right)+\left(x-\frac{a+b}{2 a b}\right) \\
& \left.\times\left(\cosh \gamma y+\frac{a+b}{\gamma} \sinh \gamma y\right)\right][W(x)]^{-1}
\end{aligned}
$$

for $x+y>0$.

For the $(2,2)$ entry $f(y)=K_{22}^{(-)}(x, y)$ satisfies

$$
\begin{aligned}
& f^{\prime \prime}(y)-(a+b) f^{\prime}(y)+a b f(y)-a b f(-y)=0, \\
& f(-x)=0, \quad f^{\prime}(-x)=a b,
\end{aligned}
$$

and an analogous calculation gives

$$
\begin{aligned}
K_{22}^{(-)}= & -a b([(a+b) / \gamma] \cosh \gamma y-\sinh \gamma x) \\
& +a b([(a+b) / \gamma] \cosh \gamma y+\sinh \gamma y) \\
& \times[-(a+b) \sinh \gamma x+\gamma \cosh \gamma x]^{-1} .
\end{aligned}
$$

For the $(1,2)$ and $(2,1)$ entries the nonhomogeneous terms in (4.4) vanish and we obtain $K_{12}^{(-)}(x, y)$ $=K_{21}^{(-1}(x, y)=0$.

To determine $v(x)$ and $p(x)$ we use (2.12). For $x=y$ the expressions for $K^{(-)}(x, y)$ reduce to

$$
\begin{aligned}
K_{11}^{(-)}(x, x)= & -a b[2 x \cosh \gamma x \\
& \left.-\left((a+b)^{2} / 2 \gamma a b\right) \sinh \gamma x\right][W(x)]^{-1}, \\
K_{22}^{\prime-1}(x, x)= & 2 a b \sinh \gamma x /(\gamma \cosh \gamma x \\
& -(a+b) \sinh \gamma x) .
\end{aligned}
$$

Checking (2.12) we have

$$
v(x)=0, \quad p(x)=K_{11}^{(-)}(x)-K_{22}^{(-)}(x), \quad x>0 .
$$

The asymptotic form of $p(x)$ is available upon retaining only the growing exponential terms in (4.7). In particular, the coefficient of $x$ in $p(x)$ vanishes and leaves $p(x) \cong 1 /(x+\sigma), x \rightarrow \infty$, where $\sigma$ is a constant.

\section{ACKNOWLEDGMENT}

The authors wish to acknowledge and thank Professor B. M. Levitan, Moscow State University, for useful conversations concerning this paper.
' K. Chadan and P. C. Sabatier, Inverse Problems in Quantum Scattering Theory (Springer, New York, 1989), 2nd ed.

${ }^{2}$ D. B. Hinton, M. Klaus, and J. K. Shaw, "Levinson's theorem and Titchmarsh-Weyl theory for a Dirac system," Proc. R. Soc. Edinburgh 109A 173 (1988).

${ }^{3}$ D. B. Hinton, M. Klaus, and J. K. Shaw, "Asymptotic phase, asymptotic modulus and Titchmarsh-Weyl coefficient for a Dirac system," J. Math. Anal. Appl. 142, 108 (1989).

${ }^{4}$ D. B. Hinton, M. Klaus, and J. K. Shaw, "High-encrgy asymptotics for the scattering matrix on the line," Inverse Prob. 5, 1049 (1989),

${ }^{5}$ D. B. Hinton, M. Klaus, and J. K. Shaw, "Series representation and asymptotics for Titchmarsh-Weyl $m$-functions," Diff. Int. Eqs. 2, 419 (1989).

${ }^{6} \mathrm{D}$. B. Hinton, M. Klaus, and J. K. Shaw, "On transformation operators and the Marchenko equation for a Dirac system," in preparation.

${ }^{7} \mathrm{~J}$. Weidmann, Spectral Theory of Ordinary Differential Operators (Springer Lecture Notes in Mathematics, Berlin, 1987), Vol. 1258.

"I. S. Frolov, "Inverse scattering problem for a Dirac system on the whole axis," Sov. Math. Dokl. 13, 1468 (1972).

${ }^{9}$ N. Asano and Y. Kato, "Non-self-adjoint Zakharov-Shabat operator with a potential of the finite asymptotic values," J. Math. Phys. 25, 570 (1984).

${ }^{10}$ The authors wish to thank Professor B. Konopelchenko, Institute of Nuclear Physics, USSR Academy of Sciences, for pointing out Ref. 9.

"V. A. Marchenko, Sturm-Liouville Operators and Applications (Birkhäuser, Basel, 1986).

${ }^{12}$ B. M. Levitan, Inverse Sturm-Liouville Problems (VNU Science, Utrecht, 1987).

${ }^{13}$ I. Kay and H. E. Moses, Inverse Scattering Papers (1955-63/Math Science Press, Brookline, MA, 1982).

${ }^{14}$ A. K. Jordan and S. Lakshmanasamy, "Inverse scattering theory applied to the design of single-mode planar optical waveguides," J. Opt. Soc. Am. A. 6, 1206 (1989).

${ }^{15}$ M. G. Gasymov and B. M. Levitan, "The inverse problem for a Dirac system, " Sov. Math. Dokl. 7, 495 (1966).

${ }^{16}$ M. G. Gasymov and B. M. Levitan, "Determination of Dirac's system from the scattering phase," Sov. Math. Dokl. 7, 543 (1966).

${ }^{17}$ B. M. Levitan and I. S. Sargsjan, Introduction to Spectral Theory, transia. tion of Mathematical Monographs, Vol. 39 (Am. Math. Soc., Providence, RI, 1975)

${ }^{18}$ M. G. Gasymov, "The inverse scattering problem for a system of Dirac equations of order $2 n$," Trans. Moscow Math. Soc. 19, 41 (1968).

${ }^{19} \mathrm{R}$. Weiss, W. Stahel, and G. Scharf, "The inverse problern of potential scattering according to the Dirac equation," Nucl. Phys. A 183, 337 (1972).

${ }^{20}$ V. E. Zakharov and A. B. Shabat, "Exact theory of $t$ wo-dimensional selffocusing and one-dimensional self-modulation of waves in nonlinear media," Sov. Phys. JETP 34, 62 (1972).

${ }^{21}$ V. E. Zakharov and A. B. Shabat, "Interactions between solitons in a stable medium," Sov. Phys. JETP 37, 823 (1973).

${ }^{22}$ H. Grosse, "New solitons connected to the Dirac equations," Phys. Rep. 134, 297 (1986).

${ }^{23} \mathrm{H}$. Grosse and G. Opelt, "Fractional charges in external field problems and the inverse scattering method," Nucl. Phys. B 285, 143 (1987).

${ }^{24}$ G. P. Bava and G. Ghione, "Inverse scattering for optical couplers: Exact solution of Marchenko equations," J. Math. Phys. 25, 1900 (1984).

${ }^{25}$ G. P. Bava, G. Ghione, and I. Maio, "Fast exact inversion of the generalized Zakharov-Shabat problem for rational scattering data: application to the synthesis of optical couplers," SIAM J. Appl. Math. 49, 489 (1988).

${ }^{26}$ D. Marcuse, Light Transmission Optics (Van Nostrand Reinhold, New York, 1972).

${ }^{27}$ L. D. Faddeev and L. A. Takhtajan, Hamiltontian Methods in the Theory of Solitons (Springer, Berlin, 1987).

${ }^{28}$ D. P. Clemence, "Low-energy scattering and Levinson's theorem for a one-dimensional Dirac equation," Inverse Prob. 5, 269 (1989).

${ }^{29} \mathrm{E}$. C. Titchmarsh, The Theory of Functions (Oxford U. P., London, 1939), 2nd ed. 